\title{
Optimization Techniques for Sparse Matrix-Vector Multiplication on GPUs
}

\author{
Marco Maggioni ${ }^{\mathrm{a}, *}$, Tanya Berger-Wolf ${ }^{\mathrm{a}}$ \\ ${ }^{a}$ Department of Computer Science, University of Illinois at Chicago \\ 851 S. Morgan, Room 1120 SEO, Chicago, IL 60607-7053, USA
}

\begin{abstract}
Sparse linear algebra is fundamental to numerous areas of applied mathematics, science and engineering. In this paper, we propose an efficient data structure named AdELL+ for optimizing the SpMV kernel on GPUs, focusing on performance bottlenecks of sparse computation. The foundation of our work is an ELL-based adaptive format which copes with matrix irregularity using balanced warps composed using a parametrized warp-balancing heuristic. We also address the intrinsic bandwidth-limited nature of SpMV with warp granularity, blocking, delta compression and nonzero unrolling, targeting both memory footprint and memory hierarchy efficiency. Finally, we introduce a novel online auto-tuning approach that uses a quality metric to predict efficient block factors and that hides preprocessing overhead with useful SpMV computation. Our experimental results show that AdELL+ achieves comparable or better performance over other state-of-the-art SpMV sparse formats proposed in academia (BCCOO) and industry (CSR+ and CSR-Adaptive). Moreover, our auto-tuning approach makes AdELL+ viable for real-world applications.
\end{abstract}

\section{(c) 2015 Published by Elsevier Ltd.}

Keywords:

SpMV, optimization, GPU, adaptive, AdELL+, blocking, compression, unrolling, auto-tuning

${ }^{*}$ Corresponding author: 1-(312)-996-8635

Email addresses: mmaggi3@uic .edu (Marco Maggioni), tanyabw@uic .edu (Tanya Berger-Wolf) 


\section{Introduction}

Sparse Matrix-Vector multiplication (SpMV) is a fundamental computational kernel (it is one of the seven dwarves [1]) and its efficiency is crucial for numerous applications in science and engineering. The widespread importance of sparse matrix computation has resulted in significant research efforts poured into implementations based on modern-day parallel computer architectures, allowing the solution of increasingly complex computational problems in areas such as linear algebra [2], convex optimization [3], data mining [4] and graph analytics [5]. In general, the research community focused on providing every possible improvements under the valid assumption that so many applications can benefit from better performance, however incremental the approach.

The need of pursuing better implementations based on Graphic Processing Units (GPUs) has been recently questioned by Davis and Chung [6]. Their argument was based on the narrowing memory bandwidth gap between CPUs and GPUs making GPU-based implementations not really worth the additional effort. It might be observed that the bandwidth gap shows a cyclic behavior, where a new fast memory technology is firstly introduced on GPUs and then adopted later by CPUs (indeed, the cycle is about to repeat with Stacked DRAM on the future NVIDIA Pascal architecture). Hence, there will always be an edge in terms of memory bandwidth from which real-world sparse linear algebra computation can benefit. On the other hand, performance-per-watt is the metric that is driving the evolution of large scale scientific computing. Manycore architectures are well-known for their power-efficiency [7]. Therefore, GPU-based implementations will continue to be a relevant research topic with solid practical applications.

Over the last decade, SpMV optimization has been extensively studied on many-core GPUs, with the aim of exploiting the available fine-grain parallelism and memory bandwidth. Despite having a fairly straightforward sequential implementation, the SpMV kernel is a pure memory bound problem due to its extremely low arithmetic intensity and potentially irregular memory patterns. As a consequence, its parallel performance is generally reported to sustain low fractions of computational peak [8]. Several hand-optimized GPU-based sparse matrix formats achieved impressive progresses targeting specific structural features of the underlying problems. However, we conjecture that the SpMV kernel can be further improved by addressing its performance bottlenecks in a more explicit way. In this paper, we propose to integrate several performance optimization techniques (each one targeting a complementary goal) into AdELL+, an efficient data structure for sparse linear algebra computation.

\subsection{Related work}

The literature on implementing SpMV on throughput-oriented manycore processors is extensive. The main focus has been on proposing and tuning novel formats to efficiently represent the sparse matrix, with the aim of optimizing memory pattern efficiency, memory footprint, load balancing and thread divergency. Bell and Garland presented perhaps the best-known work on GPU-based SpMV [9], including basic sparse matrix formats such as COO, DIA, CSR, ELL, and a new hybrid format HYB (which combines ELL and $\mathrm{COO}$ ). The vendor-tuned library cuSPARSE [10] provides a well-maintained implementation of the above formats. However, none of them is consistently superior since each has been designed to leverage a specific matrix structure. COO and CSR are suited for unstructured matrices, with COO rarely used in practice due to its space inefficiency. ELL is instead suited for vector architectures and performs well with regular structures. DIA provides a clear advantage for strongly diagonal matrices. Several derivative formats have been subsequently proposed in order to improve the baseline performance. ELL-R [11] uses an auxiliary data structure to store row lengths and avoid wasteful computation. Baskaran and Bordawekar [12] improve the CSR-based SpMV kernel by assuring the memory alignment and by caching values repeatedly accessed. Another format known as SELL [13] (and its generalization for heterogenous vectorized units SELL-C- $\sigma$ [14]) reduces the memory footprint of the basic ELL format by horizontally partitioning the original matrix into several slices.

Choi et al. [15] added the idea of blocking to CSR and ELL, creating two novel sparse formats known as BCSR and BELL. This technique is beneficial for matrices with block substructure and intrinsically reduces the memory footprint (by using indices to entire blocks instead of individual nonzero entries). Row and column reordering is another strategy than can be used to reduce matrix bandwidth and improve cache 
locality during SpMV execution [16]. However, original matrices often have inherent locality so reordering may not have any practical benefit (especially considering the preprocessing overhead). There are some works focusing on compression to reduce the matrix footprint and, consequently, improve the bandwidthlimited SpMV kernel. A bit-representation index compression scheme has been proposed by Tang et al. [17]. Another work by Xu et al. [18] focused on index compression for matrices with diagonal structure. In general, the challenge of compression techniques is the complexity of decompression. This operation becomes embedded into the SpMV kernel and should be as lightweight as possible in order to not cancel out the performance gain (which is also limited by the intrinsic matrix compressibility). Another approach to SpMV optimization is tuning the sparse format parameters (e.g. block size or slicing factor) in order to achieve the best possible performance. This is usually done by the means of auto-tuning frameworks that explore the parameter search space (usually a reasonable subset). While auto-tuning is beneficial, it also introduces additional preprocessing and benchmarking costs that should be accounted for. The success of ensemble approaches like clSpMV [19] heavily relies on auto-tuning. Since different matrix sparsity patterns can be related to specific optimizations, the clSpMV framework provides an attractive approach based on analyzing the matrix structure and appropriately selecting the best representation (or a combination of these) among an ensemble of many available GPU-based sparse formats.

The greatest challenge in SpMV optimization is to cope with the irregular matrix structure. Row-based parallelization (i.e. assigning one working thread to each row) leads to load imbalance since nonzeros are unevenly distributed across different rows. Roughly speaking, this problem can be solved by distributing threads to rows according to their computational load, a technique known as adaptivity. The first step towards the implementation of this technique was the ability to use collaborative threads to process a single row (as opposed to the row-based parallelization just described). Advanced sparse matrix formats such as ELL-T [20], SIC [21], CMRS [22] or RgCSR [23] use slightly different approaches to map a row to one or more threads in a warp. Unfortunately, this thread mapping is not flexible (i.e. each row is mapped to exactly $t$ threads, where $t$ is a parameter) hence unbalanced workloads are still problematic. A more flexible implementation was then proposed by Heller and Oberhuber [24]. However, their ArgCSR composes balanced blocks using a very simple policy that fails in the case of very skewed nonzero distributions (e.g. matrices with a single dense row). A complete (and arguably superior) adaptive sparse matrix format is provided by AdELL and its warp-balancing heuristic to compose warps [25]. More recent work on adaptivity has used two-dimensional blocking for composing balanced computation (BRC [26]) and dynamic parallelism for processing heavyweight rows (ACSR [27]).

Focusing on pure performance, the state-of-the-art in SpMV optimization is arguably BCCOO [28]. This advanced sparse matrix format is an evolution of COO based on blocking and row indices compression, where load balancing is achieved by the means of a highly-efficient segmented reduction (which, however, relies on a non-portable synchronization-free mechanism that stalls on modern AMD GPUs). BCCOO, as many other advanced sparse formats, introduces a significant preprocessing overhead for data structure generation and tuning. This set-up cost is acceptable in applications where it can be amortized over repeated SpMV iterations on the same matrix or on matrices with the exact same sparse structure. For this reason, GPU vendors focused their research on performance with lightweight preprocessing over CSR. NVIDIA Research recently released the ModernGPU library [29], a collection of advanced GPU coding approaches including CSR+. Based on segmented reduction for load balancing, CSR+ provides very promising performance with negligible preprocessing overhead. On the same note, AMD Research recently proposed CSR-Adaptive [30]. With a slightly different interpretation of adaptivity, CSR-Adaptive uses an unconventional (compared to previous work) approach to process short/medium rows (segmented reduction over nonzeros buffered in local memory).

Despite the impressive progresses achieved in SpMV optimization, we observe that no previous work has ever explicitly combined different optimization techniques in order to effectively address all the performance bottlenecks of sparse linear algebra computation. This, arguably, provides a promising approach to improve the state-of-the-art, motivating the research work presented in this paper. Our approach combines in an efficient and structured way the existing techniques to achieve maximum performance. In addition, we recognize the need to keep the preprocessing as small as possible in order to amortize the additional overhead. 


\subsection{Contribution}

The main contribution of our research is combining the leading ideas in SpMV optimization on GPUs into a lightweight, general, adaptive, efficient and high-performance method for sparse linear algebra computation. This result has been achieved by proposing AdELL+, an advanced sparse matrix format that explicitly addresses the performance bottlenecks of the SpMV kernel. Building upon our previous work $[25,31]$, we carefully composed several optimization techniques into a data structure with warp granularity that naturally suits the Single Instruction Multiple Data (SIMD) vectorization associated with many-core GPUs. AdELL+ has several new distinctive advantages that we exploit to reach a substantial performance edge over the state-of-the-art BCCOO. First, it is derived from ELL (a general sparse format well-suited for vectorization and coalesced memory access) but makes no assumption on the sparsity structure. Second, load balancing is achieved by embedding balanced warps directly into the data structure itself rather than solely relying on a (non-portable) global segmented reduction. All the collaborative operations can be then implemented using fast warp intrinsic (plus few atomics in case of heavyweight rows processed using multiple warps), achieving a portable, adaptive and efficient solution. Third, the preprocessing and tuning cost of AdELL+ is more lightweight than BCCOO thanks to the proposed online auto-tuning approach guided by a novel quality metric. In addition, AdELL+ is competitive with CSR+ and CSR-Adaptive for those applications where preprocessing cannot be easily amortized. More specifically our contributions are as follows.

- A transparent integration of complementary memory optimization techniques into AdELL [25]. We address the bandwidth-limited nature of the SpMV kernel by using a new format based on warp granularity, blocking, and delta-based index compression (to reduce the memory footprint) plus nonzero unrolling (to improve the memory hierarchy utilization). We also provide an in-depth analysis of those techniques, devising a theoretical quality metric that can be used for performance evaluation and prediction.

- A parametrized adaptive warp-balancing heuristic to implement the idea of adaptive warp-balancing. Our greedy heuristic distributes workload to hardware-level blocks (warps), with the aim to create a balanced (and efficient) AdELL+ structure. Differently from what was originally proposed [25], we process the matrix incrementally without changing the row ordering. This is beneficial for two reasons. First, we exploit the locality of the original matrix. Second, we avoid the layer of complexity associated with reordering (i.e. storing the matrix mapping). Thus, this approach provides comparable results in terms of warp-balancing with lower processing cost.

- A novel online auto-tuning approach based on the aforementioned quality metric. This metric allows a much faster evaluation of performance and a quick search of the parameter space. The underlying idea is to progressively explore the space of optimization parameters with line search while the actual SpMV kernel is doing useful computation, completely subsuming all the preprocessing overhead associated with composing AdELL+.

We evaluated floating-point performances of our approach on a benchmark suite composed of 14 regular and 6 irregular matrices from heterogenous application domains. Our experimental results on Tesla K40 showed that our proposed approach consistently achieves comparable or better performance over BCCOO (1.22x speedup on regular and 1.05x on irregular benchmarks). Those results were replicated over other state-of-the-art sparse formats (CSR+ and CSR-Adaptive) with even larger margins. The high level of optimization achieved by AdELL+ can be also proved by its effective memory bandwidth (close to the peak achievable on Tesla K40). The widespread importance of SpMV (and the massive effort poured by the research community into its optimization) makes this result greatly relevant in practice. Moreover, the proposed online auto-tuning approach makes AdELL+ directly applicable for real-world applications.

The rest of the paper is organized as follows: Section 2 reviews the existing SpMV formats and related work. In Section 3, we introduce warp-granularity into the ELL format. Section 4 describes the loop unrolling technique. Section 5 and 6 discuss two techniques to improve the memory footprint. Section 7 introduces the adaptivity technique and our warp-balancing heuristic. Section 8 puts all those techniques 
together into AdELL+. Section 9 explains how to efficiently perform autotuning. Section 10 provides a comparison with the state-of-the-art in SpMV optimization. Section 11 focuses on bandwidth and cache memory considerations. Last, Section 12 is devoted to conclusions.

\section{Background}

The SpMV is, arguably, the most important kernel in sparse matrix computations. In this paper, we specifically refer to the operation $\mathbf{y}=\mathbf{y}+\mathbf{A x}$, where $\mathbf{y}$ and $\mathbf{x}$ are dense vectors of length $n$ and $m$, respectively, while $\mathbf{A}$ is a sparse matrix of size $n \times m$. Typical matrices in applications have thousands or even millions of rows and columns with only a small fraction of nonzeros entries. Calculation of the SpMV essentially reduces to many independent dot products such as the following

$$
y_{i}=y_{i}+\sum_{\forall j: a_{i j} \in \mathbf{A}_{\mathbf{i}}} a_{i j} x_{j},
$$

where $\mathbf{A}_{\mathbf{i}}$ is a sparse row vector of the matrix $\mathbf{A}$ and $a_{i j}$ corresponds to its nonzero entries. While SpMV computation may seem intrinsically parallel, several challenges are faced in achieving high performance. First, an irregular structure may lead to an unbalanced workload with uneven nonzeros across different working threads. Such imbalance problem is even more severe in many-core GPUs since warps operate in SIMD fashion, leading to potential control divergency (e.g. a thread processing a heavyweight row that forces adjacent threads idle). Second, the bandwidth-limited nature of the SpMV kernel puts high pressure on the memory hierarchy. Each nonzero $a_{i j}$ in matrix $\mathbf{A}$ is only used once for computing the correspondent dot product, whereas the elements of the dense vector $\mathbf{x}$ may be reused across different dot products (although we expect poor locality in case of irregular structure). Roughly speaking, SpMV can be seen as a streaming computation, where performance is limited by the bandwidth at which matrix data can be streamed from memory. Moreover, modern GPUs can benefit from a cache hierarchy (usually organized on two levels) that performs best in case of aligned and coalesced memory operations (e.g. different threads in a warp accessing data within the same cache line).

\subsection{Sparse matrix characterization}

The sparse structure provides the opportunity to represent a matrix in memory by only its nonzero elements (plus additional information associated with row and column indices) as opposed to using a twodimensional dense array (which would considerably limit sizes of computed problems). In some cases, the pattern of nonzero elements is quite regular (e.g. for problems resulting from discretization on regular grids),

\begin{tabular}{|c||r|r|r|r|r|}
\hline \multirow{2}{*}{ Matrix } & \multicolumn{3}{c||}{ Dimension } & \multicolumn{1}{c|}{ Nonzeros per Row } \\
\cline { 2 - 6 } & \multicolumn{1}{|c|}{$n n z$} & \multicolumn{1}{c|}{$n$} & \multicolumn{1}{c|}{$m$} & \multicolumn{1}{c|}{$\mu$} & \multicolumn{1}{c|}{$\sigma$} \\
\hline \hline Circuit & 170998 & 170998 & 958936 & 5.61 & 4.39 \\
\hline Dense & 4000000 & 2000 & 2000 & 2000.00 & 0.00 \\
\hline Economics & 1273389 & 206500 & 206500 & 6.17 & 4.44 \\
\hline Epidemiology & 2100225 & 525825 & 525825 & 3.99 & 0.08 \\
\hline FEM/Accelerator & 2624331 & 121192 & 121192 & 21.65 & 13.79 \\
\hline FEM/Cantilever & 4007383 & 62451 & 62451 & 64.17 & 14.06 \\
\hline FEM/Harbor & 2374001 & 46835 & 46835 & 50.69 & 27.78 \\
\hline FEM/Ship & 7813404 & 140874 & 140874 & 55.46 & 11.07 \\
\hline FEM/Spheres & 6010480 & 83334 & 83334 & 72.13 & 19.08 \\
\hline Ga41As41H72 & 18488476 & 268096 & 268096 & 68.96 & 105.39 \\
\hline Protein & 4344765 & 36417 & 36417 & 119.31 & 31.86 \\
\hline QCD & 1916928 & 49152 & 49152 & 39.00 & 0.00 \\
\hline Si41Ge41H72 & 15011265 & 185639 & 185639 & 80.86 & 126.97 \\
\hline Wind Tunnel & 11634424 & 217918 & 217918 & 53.39 & 4.74 \\
\hline
\end{tabular}

Table 1. Description of the regular benchmark suite from the University of Florida Sparse Matrix Collection [32]. 


\begin{tabular}{|c||r|r|r||r|r|}
\hline \multirow{2}{*}{ Matrix } & \multicolumn{3}{|c||}{ Dimension } & \multicolumn{1}{c|}{ Nonzeros per Row } \\
\cline { 2 - 6 } & $n n z$ & $n$ & \multicolumn{1}{c|}{$m$} & \multicolumn{1}{c|}{$\mu$} & \multicolumn{1}{c|}{$\sigma$} \\
\hline \hline Circuit5M & 59524291 & 5558326 & 5558326 & 10.71 & 1356.62 \\
\hline Eu-2005 & 19235140 & 862664 & 862664 & 22.30 & 29.33 \\
\hline In-2004 & 16917053 & 1382908 & 1382908 & 12.23 & 37.23 \\
\hline LP & 11279748 & 4284 & 1092610 & 2632.99 & 4209.26 \\
\hline Mip1 & 10352819 & 66463 & 66463 & 155.77 & 350.74 \\
\hline Webbase & 3105536 & 1000005 & 1000005 & 3.11 & 25.35 \\
\hline
\end{tabular}

Table 2. Description of the irregular benchmark suite from the University of Florida Sparse Matrix Collection [32]

but the most interesting (and challenging) matrices are those whose distribution of nonzeros per row appears to be highly variable. The matrix sparsity pattern can be roughly evaluated by some simple metrics based on the number of nonzeros per row in $\mathbf{A}$. We can immediately calculate the average nonzeros per row $\mu$ and the associated standard deviation $\sigma$. Thus, we can get an approximate idea of the nonzero distribution (and, consequently, a sense of the load balancing) by looking at the minimum, median and maximum nonzeros per row along with its binned histogram.

Here we introduce the characterization of the benchmark suite used to provide a comprehensive evaluation of our work. All the sparse matrices (except a synthetically generated dense one) are downloadable from the well-known University of Florida Sparse Matrix Collection [32] as Matrix Market files [33]. As suggested by Langr and Tvrdik [34] in their evaluation criteria for sparse matrix formats, we provide a fair comparison by choosing relatively large matrices that have been widely adopted in previous SpMV optimization research $[8,9,13-15,19,21,25,26,28,30,31]$. This benchmark suite contains 20 sparse matrices of heterogenous sizes, structures, number of overall nonzeros (i.e. nnz) and application domains. We categorize them as regular (14 matrices in Table 1) and irregular (6 matrices in Table 2), based on the coefficient of variation $\frac{\mu}{\sigma}$ as well as on the distribution of the nonzeros per row.

The adopted classification is more evident from Tables 3 and 4. Each irregular matrix has a large range [min, $\max ]$ and its associated histogram (built with uniform bins within the range $[0, \max ]$ ) highlights a skewed distribution of nonzeros per row. Due to the large range, rows falling in the last bins account for a significant percentage of the total nonzeros (e.g. $13 \%$ of total nonzeros in Circuit5M are associated with rows falling in the last bin). Clearly, this sparsity pattern is hard to parallelize. On the other hand, regular matrices distribute their rows more freely along the range of nonzeros per row. Some benchmarks, such

\begin{tabular}{|c|c|c|c|c|c|c|c|c|c|c|c|c|c|}
\hline \multirow[b]{2}{*}{ Matrix } & \multicolumn{3}{|c|}{ Nonzeros per Row } & \multirow{2}{*}{\multicolumn{10}{|c|}{$\begin{array}{c}\text { Binned } \\
\text { Histogram }\end{array}$}} \\
\hline & $\min$ & med & $\max$ & & & & & & & & & & \\
\hline Circuit & 1 & 5 & 353 & & & & & & & & & & \\
\hline Dense & 2000 & 2000 & 2000 & & & & & & & & & & \\
\hline Economics & 1 & 5 & 44 & & & & & & & & & & \\
\hline Epidemiology & 2 & 4 & 4 & & & & & & & & & & \\
\hline FEM/Accelerator & 0 & 23 & 81 & & & & & & & & & & \\
\hline FEM/Cantilever & 1 & 74 & 78 & & & & & & & & & & \\
\hline FEM/Harbor & 4 & 46 & 145 & & & & & & & & & & \\
\hline FEM/Ship & 24 & 54 & 102 & & & & & & & & & & \\
\hline FEM/Spheres & 1 & 81 & 81 & & & & & & & & & & \\
\hline Ga41As41H72 & 18 & 37 & 702 & & & & & & & & & & \\
\hline Protein & 18 & 123 & 204 & & & & & & & & & & \\
\hline QCD & 39 & 39 & 39 & & & & & & & & & & \\
\hline Si41Ge41H72 & 13 & 37 & 662 & & & & & & & & & & \\
\hline Wind Tunnel & 2 & 54 & 180 & & & & & & & & & & \\
\hline \multicolumn{4}{|c|}{ Legend (Nonzero Density) } & $\leq 10 \%$ & $\leq 20 \%$ & $\leq 30 \%$ & $\leq 40 \%$ & $\leq 50 \%$ & $\leq 60 \%$ & $\leq 70 \%$ & $\leq 80 \%$ & $\leq 90 \%$ & $\leq 100 \%$ \\
\hline
\end{tabular}

Table 3. The distribution of nonzeros per row in the regular matrices. The bins are uniformly spaced in the range [0, max] and highlighted according to their density (zero is equivalent to absence of nonzeros in the bin). 


\begin{tabular}{|c|c|c|c|c|c|c|c|c|c|c|c|c|c|}
\hline \multirow[b]{2}{*}{ Matrix } & \multicolumn{3}{|c|}{ Nonzeros per Row } & \multirow{2}{*}{\multicolumn{10}{|c|}{$\begin{array}{c}\text { Binned } \\
\text { Histogram }\end{array}$}} \\
\hline & $\min$ & med & $\max$ & & & & & & & & & & \\
\hline Circuit5M & 1 & 5 & 1290501 & & & & & & & & & & \\
\hline Eu-2005 & 0 & 16 & 6985 & & & & & & & & & & \\
\hline In-2004 & 0 & 5 & 7753 & & & & & & & & & & \\
\hline LP & 1 & 1158 & 56181 & & & & & & & & & & \\
\hline Mip1 & 4 & 40 & 66395 & & & & & & & & & & \\
\hline Webbase & 1 & 2 & 4700 & & & & & & & & & & \\
\hline \multicolumn{4}{|c|}{ Legend (Nonzero Density) } & $\leq 10 \%$ & $\leq 20 \%$ & $\leq 30 \%$ & $\leq 40 \%$ & $\leq 50 \%$ & $\leq 60 \%$ & $\leq 70 \%$ & $\leq 80 \%$ & $\leq 90 \%$ & $\leq 100 \%$ \\
\hline
\end{tabular}

Table 4. The distribution of nonzeros per row in the irregular matrices.

as QCD, are perfectly balanced since $\sigma=0$. Some other benchmarks, such as Circuit or Ga41As41H72, still appear skewed but their smaller range (compared with irregular matrices) makes the computing load associated with long row less severe.

\subsection{Basic sparse matrix formats}

In this subsection, we provide a brief description of the ELL sparse matrix representation (and its GPUbased SpMV kernel) as the necessary foundation to understand the remaining of this paper. ELL is a sparse format particularly well-suited to SIMD vectorized architectures. Its basic idea is to compress the sparse $n \times m$ matrix using a dense $n \times k$ data structure, where $k$ corresponds to max (the maximum number of nonzeros per row). As shown by Figure 1, the sparse matrix $\mathbf{A}$ is stored in memory by the means of two $4 \times 3$ dense arrays Value[] and Column [] (row indices remain implicit as in a dense matrix). Hence, zeropadding is necessary for rows with less than $k=3$ elements. Indeed, the memory footprint associated with strictly depends by the matrix regularity. Whenever rows approximately have the same number of nonzeros, ELL can be as efficient as CSR. On the other hand, skewed distributions may lead to a unfeasible memory footprint (i.e. as large as the dense matrix). Similarly to CSR, ELL works at the granularity of thread per row. However, its memory layout is optimized for coalesced access by adopting column-major ordering.

Figure 2 provides a detailed view on how data are stored in memory for efficiency. Due to column-major ordering, a vectorized warp-level instruction (for the sake of simplicity $w=4$ ) can process a batch of $w$ distinct nonzeros (e.g. [a,d,f,h]) by coalescing the loading of adjacent memory addresses. Given a matrix of arbitrary size, it may be also necessary to add few padding rows at the bottom of the two $n \times k$ arrays in order to guarantee $w$-alignment (indeed, this optimization is a consequence of how GPU memory hierarchy is designed). In other words, we round $n$ to the next multiple of $w\left(\right.$ i.e. $\left.n^{\prime}=\left\lceil\frac{n}{w}\right\rceil \cdot w\right)$. ELL certainly achieves good performance on regular structures (i.e. with an equal number of nonzeros on each row). On the other hand, irregular matrices inevitably lead to memory footprint inefficiency and waste of computation

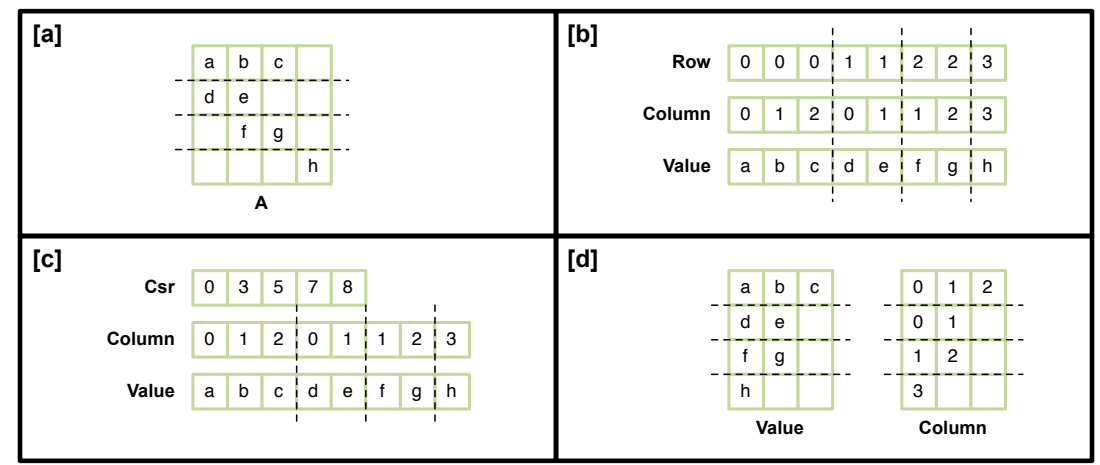

Fig. 1. Sparse matrix A [a] represented as COO [b], CSR [c] and ELL [d]. 


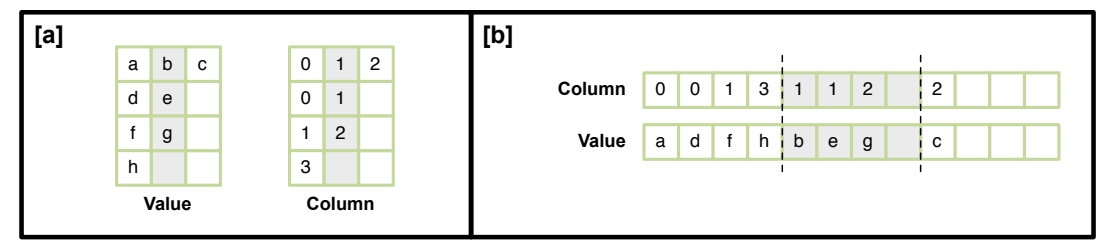

Fig. 2. ELL sparse format [a] and its column-major memory layout [b].

(i.e. short rows make their thread idle for most of the time). We can quantify efficiency as

$$
e^{E L L}=\frac{\mathrm{nnz}}{n^{\prime} \cdot k},
$$

where $n n z$ is the overall number of nonzeros in $\mathbf{A}$ and $e^{E L L}$ is a $[0,1]$ metric that measures the portion of actual nonzeros in Value[] and Column[] (intuitively, $1-e^{E L L}$ corresponds to padding).

Using ELL as reference sparse format, we can provide a roofline-type [35] performance model to characterize the SpMV kernel as bandwidth-limited. Assuming no latency effect (due to an effective hardware multithreading) and infinitely fast caches, the arithmetic intensity $i$ (the ratio between performed floatingpoint operations and bytes moved from/to memory) associated with each nonzero can be calculated as

$$
i=\left(\frac{2}{m_{\mathbf{A}}+m_{\mathbf{x}}+m_{\mathbf{y}}}\right) \frac{\text { Flops }}{\text { Byte }} .
$$

Here the factor 2 comes from the dot product (i.e. floating-point multiply and add), $m_{\mathbf{A}}$ accounts for reading the nonzero value and column index from $\mathbf{A}, m_{\mathbf{x}}$ is the traffic incurred by partially indirect access to vector $\mathbf{x}$, and $m_{\mathbf{y}}$ is the data volume for updating the result $\mathbf{y}$. Assuming single-precision and four-byte integer indices, each nonzero is represented by $m_{\mathbf{A}}=4+4$ bytes. However, we should also take into account the efficiency $e^{E L L}$ itself (which may increase the effective amount of loaded data). The effect of the memory hierarchy on accessing vector $\mathbf{x}$ is characterized by a factor $\alpha \in[0,1]$. In the worst case scenario, every access causes a cache miss so $\alpha=1$. In the ideal situation, we only account for cold misses (i.e. those necessary to fetch $\mathbf{x}$ from global memory) so each cache element will be reused a number of times equal to the average number of nonzeros per column ( $\alpha=1 / \mu$ for square matrices). The cost of updating $\mathbf{y}$ elements (one read and one write) is instead amortized on $\mu$ nonzeros when using a thread to process a row. All those concepts are substituted in formula (3) to achieve the following expression

$$
i=\left(\frac{2}{(4+4) / e^{E L L}+4 \alpha+2 \cdot 4 / \mu}\right) \leq 0.250\left[\frac{\text { Flops }}{\text { Byte }}\right] \text {. }
$$

Let us assume the best case where $e^{E L L}=1, a \approx 0$ and $\mu$ is large. We can then derive an upper bound of 0.250 for a single-precision arithmetic intensity. Let us now consider the specific Tesla K40 GPU based on Kepler architecture [36] used in our experimental section. Given a $207.5 \mathrm{~GB} / \mathrm{s}$ measured peak bandwidth with ECC disabled (as opposed to $288.4 \mathrm{~GB} / \mathrm{s}$ theoretical), the upper bound on SpMV performance measured in FLoating-points Operation Per Second (or FLOPS) is 51.88 GFLOPS. This upper bound is only a small fraction of the 4.29 TFLOPS Tesla K40 theoretical computational peak, proving that SpMV is a bandwidthlimited kernel due to its low arithmetic intensity. We can repeat the same analysis for double-precision computation obtaining

$$
i=\left(\frac{2}{(8+4) / e^{E L L}+8 \alpha+2 \cdot 8 / \mu}\right) \leq 0.167\left[\frac{\text { Flops }}{\text { Byte }}\right],
$$

which corresponds to an even more capped upper bound of 34.58 GFLOPS, still a small fraction of the double-precision 1.43 TFLOPS Tesla K40 theoretical peak. Until here, we assumed an ideal cache behavior. However, in reality, the irregular memory access to the dense vector $\mathbf{x}$ could hamper performance, especially 
in case of scattered accesses. Despite being optimized for throughput, modern GPU architectures provide a cache hierarchy composed by a fully coherent L2 cache and by several local memories, each one associated with a Streaming Multiprocessor (or SMX on Kepler architecture). These latter are further divided into shared memory (i.e. a software-managed cache) and texture memory (i.e. a hardware-managed read-only cache). Despite being significantly smaller than their CPU counterparts, this cache hierarchy is a fairly efficient (and effortless) way to deal with irregularity. According to our computational experiment, L2 cache has an average hit rate of $63.77 \%$, whereas read-only texture can improve the performance by an average factor of 1.26x. On the other hand, shared memory (and its small capacity) is not useful for efficiently caching the access pattern to the dense vector $\mathbf{x}$.

\section{Improving ELL with warp granularity}

A common optimization pattern in GPU computing consists of tailoring both the data structure and the computation around the SIMD width (i.e. warp size). On the one hand, this provides an easy way to guarantee coalesced and aligned memory accesses. On the other hand, computation that involves only threads in a warp has a better performance due to its ability of exploiting implicit lockstep execution for synchronization rather than explicit primitives [37]. Similarly, collective operations between threads can be efficiently performed by building upon warp intrinsics (e.g. shuffle instruction) rather than relying on local/shared or global memory. Inspired by this ideas, we have proposed an optimized sparse format known as WELL (Warp-grained ELL) [38]. WELL partitions the matrix into (warp-sized) slices and represents them with local ELL data structures, leading to a fundamental advantage in terms of the overall memory footprint. Intuitively, the dimension size $k_{i}$ of each slice depends on the longest row in the warp, rather than a global $k$. WELL requires two additional vectors $K[]$ and Offset [] of dimension $n_{w}=\left\lceil\frac{n}{w}\right\rceil$ (the number of warps) and $n_{w}+1 . K[]$ is used to keep track of local $k_{i}$, whereas Offset[] is used to identify the incremental starting location of each local ELL structure within Value[] and Column [].

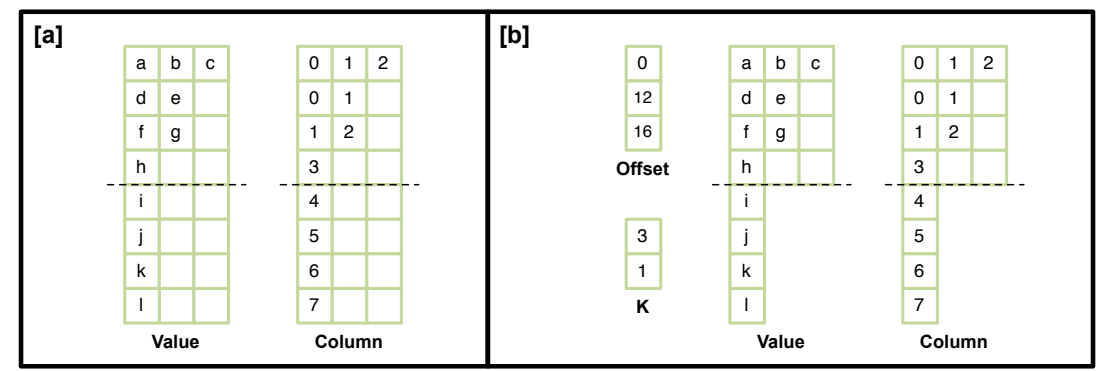

Fig. 3. An arbitrary matrix represented as ELL [a] and composed by two warps $(w=4)$. WELL [b] provide a more efficient representation with an obvious memory footprint improvement from $24(8 \times 3)$ to 16 entries (in general, we can assume $K[]$ and Offset [] to have negligible contribution since $\left.n_{w} \ll n \ll n n z\right)$.

Figure 3 shows an example of WELL and depicts the advantage of using WELL over ELL. The memory footprint improvement can be quantified by updating the efficiency formula (2) as

$$
e^{W E L L}=\frac{\mathrm{nnz}}{\sum_{i=1}^{n_{w}} w \cdot k_{i}}=\frac{\mathrm{nnz}}{\text { Offset }\left[n_{w}\right]}
$$

where the summation at the denominator takes into account each local ELL contribution to the overall WELL memory footprint. We evaluated $e^{W E L L}$ for our regular benchmark suite using both (2) and (6), obtaining the results in Table 5. Using warp granularity, WELL substantially improves over $e^{E L L}$ by a $4.73 \mathrm{x}$ factor (calculated using harmonic mean), leading to immediate benefits in terms of memory footprint and, hence, the overall SpMV computation. Table 6 shows the results for the other irregular matrices. The extremely low $e^{E L L}$ associated with ELL is alone sufficient to justify that WELL is the only viable option between the two sparse formats to represent irregular matrices (i.e. ELL is so inefficient that some matrices do not even fit in memory). 


\begin{tabular}{|c||c|c|}
\hline \multicolumn{1}{|c||}{\multirow{2}{*}{ Matrix }} & \multicolumn{2}{c|}{ Efficiency } \\
\cline { 2 - 3 } & ELL & WELL \\
\hline \hline Circuit & 0.016 & 0.398 \\
\hline Dense & 0.992 & 0.992 \\
\hline Economics & 0.140 & 0.275 \\
\hline Epidemiology & 0.998 & 0.999 \\
\hline FEM/Accelerator & 0.267 & 0.840 \\
\hline FEM/Cantilever & 0.823 & 0.881 \\
\hline FEM/Harbor & 0.349 & 0.663 \\
\hline FEM/Ship & 0.544 & 0.846 \\
\hline FEM/Spheres & 0.890 & 0.930 \\
\hline Ga41As41H72 & 0.098 & 0.364 \\
\hline Protein & 0.584 & 0.796 \\
\hline QCD & 1.000 & 1.000 \\
\hline Si41Ge41H72 & 0.122 & 0.410 \\
\hline Wind Tunnel & 0.297 & 0.982 \\
\hline \multicolumn{2}{|c}{}
\end{tabular}

Table 5. Efficiency of ELL versus WELL for the regular benchmarks.

\begin{tabular}{|c||c|c|}
\hline \multirow{2}{*}{ Matrix } & \multicolumn{2}{c|}{ Efficiency } \\
\cline { 2 - 3 } & ELL & WELL \\
\hline \hline Circuit5M & 0.000 & 0.247 \\
\hline Eu-2005 & 0.003 & 0.457 \\
\hline In-2004 & 0.002 & 0.367 \\
\hline LP & 0.047 & 0.220 \\
\hline Mip1 & 0.002 & 0.698 \\
\hline Webbase & 0.001 & 0.316 \\
\hline
\end{tabular}

Table 6. Efficiency of ELL versus WELL for the irregular benchmarks.

WELL has been designed to promote efficient SIMD vectorization at warp level. Intuitively, each local ELL structure is mapped to a warp that executes independently by any other warp in the SpMV kernel. Moreover, there is a conceptual analogy between threads in a warp and SIMD lanes (it is possible to use these two terms interchangeably in the context of warp computation). It might be argued that WELL memory layout intrinsically guarantees coalescing and alignment. In fact, each local ELL structure is still stored using column-major ordering for coalescing. Moreover, each local ELL structure has size $w \times k_{i}$ such that each and every offset in Offset[] is $w$-aligned. First, we do not apply local rearranging in order to avoid the extra layer of complexity (benefiting from the intrinsic locality of the original matrix). Second, we use texture (read-only) cache to map the vector $\mathbf{x}$. Specifically, each memory access to $\mathbf{x}$ will pass through a separate 48KB hardware-managed cache. Differently from the coherent L2 cache, this read-only memory is local to each SMX and cannot be polluted by the nonzeros streamed from global memory into the GPU during the SpMV kernel. Third, we let the padding zeros to be processed the same way as the rest of the nonzeros in the matrix. This additional arithmetic performs the same $0 \cdot x_{0}$ operation over and over, not introducing overhead due to the lockstep execution. Similarly, repeated memory accesses to $x_{0}$ are cheap due to caching. In other words, we gain in compile efficiency producing a control divergence free code and maintaining a uniform data structure for the entire matrix.

WELL is very similar to SELL [13] but its implementation of warp granularity goes beyond the simple choice of slices matching the warp size ( $w=32$ for Kepler architecture). GPU programming models such as CUDA [39] or OpenCL [40] logically organize threads into blocks that are mapped to vectorized units (SMXs) and then broken down to warps for the actual execution on the GPU hardware. It is well-known that block size $b$ must be chosen carefully to not undermine performance. The key difference between SELL and WELL is that the former binds slice size $s$ to block size $b$. Hence, warp granularity $s=w$ (which is the best in terms of $e^{W E L L}$ ) provides a poor choice in terms of block size (i.e. $b=w$ achieves very low utilization of the GPU hardware architecture). On the other hand, WELL implements warp granularity by 
decoupling $s$ from $b$. In other words, we are able to launch SpMV kernels with optimal $b$ while the data structure, as well as the actual computation, can still take advantage of warp granularity. In addition, WELL has a synchronization free execution whereas SELL blocks may need primitives to synchronize their warps.

\section{Unrolling nonzeros to optimize memory hierarchy efficiency}

Loop unrolling is a well-known technique in compiler optimization used to expose Instruction Level Parallelism (ILP) or to reduce the overhead associated with the loop control structure. Murthy et al. [41] provide an in-depth analysis of loop unrolling in the context of GPU programming. In general, the technique is transparently applied to optimize the execution of short loops with a fixed number of iterations $(e . g$. when processing a dense $2 \times 2$ matrix subblock). However, loop unrolling can be also useful in the context of bandwidth-limited kernels such as SpMV. Let us assume to have a loop where data (e.g. nonzeros) are loaded from memory and processed at each iteration. Memory transactions will be issued in a serialized way leading to potential memory latency inefficiency. On the other hand, unrolling allows to issue multiple independent memory transactions per iteration. This scenario clearly facilitates latency hiding. Indeed, those memory transactions are served simultaneously, improving the overall bandwidth utilization. Hence, loop unrolling is able to optimize the GPU memory hierarchy efficiency by increasing the number of on-the-fly memory transactions.

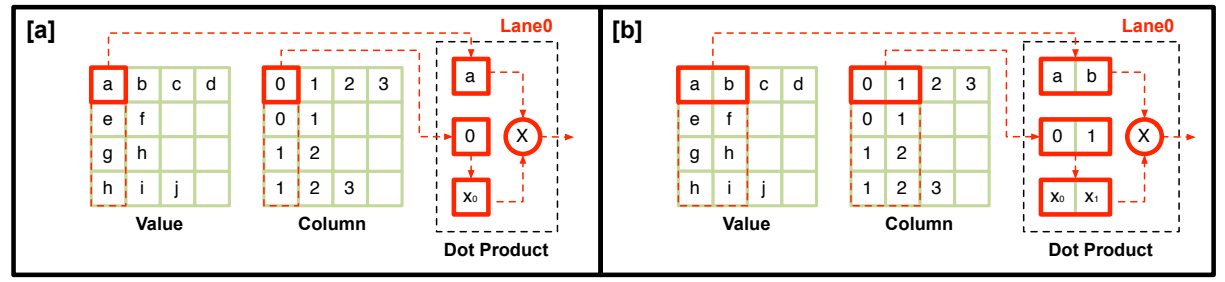

Fig. 4. Processing nonzeros without unrolling [a] and with $2 x$ unrolling [b]. The example focuses on a single warp taken from an arbitrary sparse format with warp granularity (e.g. WELL). In red, we schematized the local register buffering and the dot product operation. As we can observed, 2x unrolling needs only 2 iterations (as opposed to 4 ) to process lane 0.

Figure 4 shows the unrolling technique applied to the SpMV kernel. Without unrolling, we have a loop that iterates $k_{i}$ times over the nonzeros. For each iteration, we load both the nonzero value and column from matrix $\mathbf{A}$, we perform an indirect access to vector $\mathbf{x}$ and then we accumulate the dot product (i.e. multiply and add). Let us consider lane 0 that processes the first row. During the first iteration, value $a$ and column 0 are loaded into two local registers, $x_{0}$ is fetched into another local register and then $a \cdot x_{0}$ is computed. With unrolling, we aggressively buffer multiple nonzeros (and $\mathbf{x}$ elements) into local registers at each loop iteration (e.g. lane 0 buffers $[a, b],[0,1]$ and then $\left[x_{0}, x_{1}\right]$ ). Therefore, this technique (that we call nonzero unrolling) allows to issue multiple memory transactions simultaneously, improving bandwidth utilization and latency hiding. The overall number of iterations $k_{i}$ may not be a multiple of the loop unrolling factor $l u$. In that case, buffering will simply pad the buffers with few zeros during the last iteration. This approach clearly promotes code regularity. In fact, the added padding does not change the way in which buffers are processed (similarly, it does not affect the result correctness).

\subsection{Empirical evaluation}

Here we take time to explain the protocol for all our computational experiments (including those that will be presented in the next sections). Those were performed on a single NVIDIA Tesla K40 (2880@745MHz CUDA cores) equipped with 12GB@3004MHz ECC GDDR5 memory. We implemented all the necessary GPU-based SpMV kernels using CUDA (nvcc compiler version 6.5), and we measured the average performance in GFLOPS over 100 repetitions without considering additional overhead (e.g. moving the sparse matrix from GPU to GPU). We also disabled the Tesla K40 ECC feature and we used the 48KB texture(read-only) memory for caching vector $\mathbf{x}$. Our first goal is to provide an empirical analysis of the 


\begin{tabular}{|c||r|r|r|r|r||r|r|r|r|r|}
\hline \multirow{2}{*}{ Matrix } & \multicolumn{10}{|c|}{ Performance [GFLOPS] } \\
\cline { 2 - 11 } & $1 \mathrm{x}$ & $2 \mathrm{x}$ & $4 \mathrm{x}$ & $8 \mathrm{x}$ & $16 \mathrm{x}$ & $1 \mathrm{x}$ & $2 \mathrm{x}$ & $4 \mathrm{x}$ & \multicolumn{1}{c|}{$8 \mathrm{x}$} & $16 \mathrm{x}$ \\
\hline \hline Circuit & 4.503 & 5.752 & 9.091 & 11.273 & $\mathbf{1 2 . 0 0 7}$ & 4.138 & 5.003 & 7.849 & 8.583 & $\mathbf{9 . 2 2 9}$ \\
\hline Dense & 4.133 & 5.463 & 10.283 & 14.722 & $\mathbf{1 6 . 1 6 3}$ & 4.020 & 5.212 & 12.239 & 14.060 & $\mathbf{1 5 . 5 2 6}$ \\
\hline Economics & 8.783 & 10.484 & 12.746 & $\mathbf{1 3 . 3 3 3}$ & 13.122 & 7.354 & 7.996 & 8.978 & $\mathbf{9 . 0 4 0}$ & 8.786 \\
\hline Epidemiology & 31.828 & 35.049 & $\mathbf{3 8 . 9 4 5}$ & 38.568 & 26.441 & 24.341 & 24.701 & $\mathbf{2 5 . 9 9 1}$ & 24.487 & 17.110 \\
\hline FEM/Accelerator & 26.837 & 32.231 & 36.246 & $\mathbf{3 8 . 2 7 1}$ & 37.987 & 21.827 & 24.481 & 24.734 & 25.389 & $\mathbf{2 5 . 5 7 2}$ \\
\hline FEM/Cantilever & 28.763 & 33.938 & 41.716 & 42.804 & $\mathbf{4 4 . 2 5 3}$ & 23.434 & 25.754 & 28.687 & 30.096 & $\mathbf{3 0 . 4 7 5}$ \\
\hline FEM/Harbor & 19.179 & 22.868 & 29.796 & 31.136 & $\mathbf{3 1 . 3 8 2}$ & 16.606 & 18.420 & 21.532 & 21.746 & $\mathbf{2 1 . 9 8 9}$ \\
\hline FEM/Ship & 31.516 & 36.943 & 43.662 & 44.148 & $\mathbf{4 4 . 1 5 9}$ & 25.967 & 27.538 & 29.232 & 29.721 & $\mathbf{2 9 . 7 2 8}$ \\
\hline FEM/Spheres & 35.684 & 41.001 & 47.739 & 47.736 & $\mathbf{4 8 . 0 0 5}$ & 29.045 & 30.477 & 31.298 & 32.032 & $\mathbf{3 2 . 5 5 5}$ \\
\hline Ga41As41H72 & 12.015 & 14.550 & 18.115 & 18.684 & $\mathbf{1 9 . 2 6 8}$ & 10.353 & 11.425 & 11.901 & 12.469 & $\mathbf{1 2 . 8 9 8}$ \\
\hline Protein & 21.907 & 26.030 & 34.842 & 37.720 & $\mathbf{4 0 . 7 8 3}$ & 19.121 & 21.457 & 25.349 & $\mathbf{2 7 . 3 9 1}$ & 26.713 \\
\hline QCD & 34.674 & 40.032 & 46.969 & $\mathbf{4 7 . 9 2 9}$ & 47.816 & 29.046 & 30.843 & 31.635 & $\mathbf{3 2 . 6 6 1}$ & 32.044 \\
\hline Si41Ge41H72 & 13.491 & 16.219 & 20.230 & 20.670 & $\mathbf{2 1 . 4 0 6}$ & 11.594 & 12.672 & 13.748 & 13.884 & $\mathbf{1 4 . 3 4 1}$ \\
\hline Wind Tunnel & 32.003 & 36.096 & 44.783 & 47.784 & $\mathbf{4 8 . 7 1 3}$ & 26.777 & 28.866 & 32.029 & 33.089 & $\mathbf{3 3 . 2 5 4}$ \\
\hline \hline & \multicolumn{9}{|c||c|c|c|}{ Single Precision } \\
\hline
\end{tabular}

Table 7. Performance results for the nonzero unrolling with WELL on the regular benchmarks.

effectiveness of the proposed nonzero unrolling technique in terms of performance. Table 7 reports the SpMV performance on the regular benchmark suite using WELL kernels with different $l u$ factors. These results can be generalized as long as warp granularity holds. Not surprisingly, higher unrolling factors lead to better performance due to a better utilization of the GPU memory hierarchy. The best result (highlighted in green) is usually associated with 16x unrolling (i.e. buffering and processing 16 nonzeros for each iteration). Some of the matrices achieve their best with smaller $l u$ due to their low $\mu$ that makes higher order buffering unnecessary (e.g. Epidemiology has $\mu=3.99$ so it achieves its best with $l u=4$ ). Over regular matrices, nonzero unrolling can generally provide a performance speedup (calculated using harmonic mean) of 1.98x for single-precision calculation and 1.60x for double-precision calculation. The smaller speedup in double-precision is associated with a larger memory footprint that intrinsically provides a more optimized baseline and, hence, less room for optimization. Last, we also notice that the benchmark Dense obtains relatively low performance despite being perfectly regular. This is due to poor GPU hardware utilization. In fact, the $n=4000$ rows are mapped to only $n_{w}=125$ warps, a small portion of the 960 warps that Tesla K40 can manage. Table 8 reports the analysis on irregular matrices. As expected, the baseline performance without unrolling is generally poor compared to the regular benchmarks (for which WELL is better suited). On the other hand, there is a substantial performance improvement (approximatively $4 \mathrm{x}$ for both single and double precision) achieved by choosing $l u=16$. Indeed, a large unrolling factor provides a more efficient processing for skewed warps (i.e. those containing very irregular rows).

\begin{tabular}{|c|c|c|c|c|c|c|c|c|c|c|}
\hline \multirow[b]{2}{*}{ Matrix } & \multicolumn{10}{|c|}{ Performance [GFLOPS] } \\
\hline & $1 \mathrm{x}$ & $2 \mathrm{x}$ & $4 \mathrm{x}$ & $8 \mathrm{x}$ & $16 \mathrm{x}$ & $1 \mathrm{x}$ & $2 \mathrm{x}$ & $4 \mathrm{x}$ & $8 \mathrm{x}$ & $16 \mathrm{x}$ \\
\hline Circuit5M & 0.095 & 0.126 & 0.243 & $\overline{0.357}$ & 0.393 & 0.093 & 0.122 & $\overline{0.290}$ & 0.350 & $\overline{\mathbf{0 . 3 8 3}}$ \\
\hline Eu-2005 & 4.590 & 5.911 & 9.750 & 12.400 & 13.094 & 4.327 & 5.380 & 8.939 & 9.832 & 10.641 \\
\hline In-2004 & 4.080 & 5.251 & 8.721 & 11.112 & 11.988 & 3.869 & 4.855 & 8.000 & 8.897 & 10.223 \\
\hline LP & 0.407 & 0.545 & 1.038 & 1.499 & 1.643 & 0.399 & 0.526 & 1.193 & 1.426 & 1.568 \\
\hline Mip1 & 0.322 & 0.427 & 0.817 & 1.197 & 1.307 & 0.314 & 0.412 & 0.972 & 1.172 & 1.282 \\
\hline \multirow[t]{2}{*}{ Webbase } & 1.322 & 1.749 & 3.195 & 4.378 & 4.880 & 1.282 & 1.662 & 3.366 & 3.930 & 4.529 \\
\hline & \multicolumn{5}{|c|}{ Single Precision } & \multicolumn{5}{|c|}{ Double Precision } \\
\hline
\end{tabular}

Table 8. Performance results for the nonzero unrolling with WELL on the irregular benchmarks.

There are additional architectural factors that should be taken into consideration while predicting the best unrolling factor. As general guideline for performance, SMX occupancy (i.e. the fraction of active threads on a SMX) [39] should be maximized by taking into account constraints such as registers per thread 
(or shared memory usage) and by choosing an appropriate block size $b$. Given an arbitrary GPU kernel, we immediately know the amount of registers per thread $r$. Moreover, we are able to evaluate how $r$ will limit the number of active blocks and, hence, SMX occupancy. For example, $r=32$ does not impose any register-related limit. On the other hand, $r=128$ and $b=96$ (i.e. a multiple of warp size) imposes a limit of 5 active blocks (each one using 12288 registers out of the 65536 available on each SMX) achieving a 0.234 occupancy. By selecting block size $b=64$, the imposed limit raises to 8 with a 0.250 occupancy. Similarly, $b=32$ corresponds to 16 active blocks with the same 0.250 occupancy. As we can see, the selection of optimal block size $b$ is a convoluted tradeoff. However, we tackle this problem using a simple but effective exhaustive approach. We test all the warp multiples $b=i \cdot w$ such that $i \in\{1,2, \ldots, 32\}$ and we choose the smallest $b$ that leads to highest SMX occupancy (in general, small blocks provide a better turnover). Referring to the example just presented, we choose $b=32$ over $b=\{64,128,256\}$ despite all the options have a 0.250 occupancy. As an aside, this selection approach has been used for tuning $b$ in all our tests.

\begin{tabular}{|c||c|c|c||c||c|c|c|}
\hline $\begin{array}{c}\text { Unrolling } \\
\text { Factor }\end{array}$ & $\begin{array}{c}\text { Registers } \\
r\end{array}$ & $\begin{array}{c}\text { Block } \\
b\end{array}$ & $\begin{array}{c}\text { SMX } \\
\text { Occupancy }\end{array}$ & $\begin{array}{c}\text { Unrolling } \\
\text { Factor }\end{array}$ & $\begin{array}{c}\text { CUDA } \\
r\end{array}$ & $\begin{array}{c}\text { CUDA } \\
b\end{array}$ & $\begin{array}{c}\text { SMX } \\
\text { Occupancy }\end{array}$ \\
\hline \hline $1 \mathrm{x}$ & 15 & 128 & 1.000 & $1 \mathrm{x}$ & 18 & 128 & 1.000 \\
\hline $2 \mathrm{x}$ & 19 & 128 & 1.000 & $2 \mathrm{x}$ & 22 & 128 & 1.000 \\
\hline $4 \mathrm{x}$ & 23 & 128 & 1.000 & $4 \mathrm{x}$ & 32 & 128 & 1.000 \\
\hline $8 \mathrm{x}$ & 32 & 128 & 1.000 & $8 \mathrm{x}$ & 48 & 128 & 0.625 \\
\hline $16 \mathrm{x}$ & 47 & 64 & 0.625 & $16 \mathrm{x}$ & 79 & 64 & 0.375 \\
\hline \hline
\end{tabular}

Table 9. SMX occupancy of SpMV kernels for WELL.

Table 9 reports registers and SMX occupancy for all the SpMV kernels that we presented so far. As we can see, buffering with large unrolling factors involves more complex code and, hence, a higher number of registers. This highlights the fact that register pressure is the limiting factor for loop unrolling, not just for SMX occupancy. The compiler has the ability to minimize the number of registers needed to implement a kernel. However, some very complex codes may cause register spilling (i.e. some registers are moved into local memory). In general, this has a very detrimental effect on performance and should be avoided as much as possible. Hence, it is not uncommon for complex kernel to achieve better performance with smaller loop unrolling factors $(e . g . l u=\{2,4\})$ where register spilling is avoided. So far, we presented how to design the data layout in order to promote efficient SIMD vectorization and how to process nonzeros in order to optimize the memory hierarchy efficiency. In the next sections, we will introduce two techniques targeted for mitigating the bandwidth-limited nature of the SpMV kernel.

\section{Exploiting dense substructures with blocking}

Dense substructures are inherently present in many sparse matrices such as those derived from partial differential equation models, providing an opportunity to further optimize the performance. Blocking is a technique similar to compression that allows to leverage dense substructure to reduce the memory footprint associated with indexing. The idea is to extract a blocked matrix from the original one and store nonzero blocks (rather than single nonzero entries). Blocking can be applied to WELL as described by Figure 5. Given the sparse matrix A with a dense substructure, its WELL representation needs 32 elements for Value [] and Column[]. Moreover, the data structure spans two warps $(w=4)$. Given the blocked matrix $\mathbf{B}$ extracted from A, we can build WELL using [2 $\times 2$ ] nonzero blocks rather than single nonzeros. We can immediately notice how this new WELL structure has 8 (block) elements rather than 32. As a result, the Column [] structure is more compact, leading to an overall space savings. In other words, each [ $2 \times 2]$ block needs to store only a single column index corresponding to subelement $[0,0]$ (everything else can be directly derived from there). We can also notice that now WELL spans a single warp with 4 lanes that process two blocks each. In other words, now WELL works at block granularity, processing multiple rows simultaneously depending on the blocking factor ( 2 in the given example). 


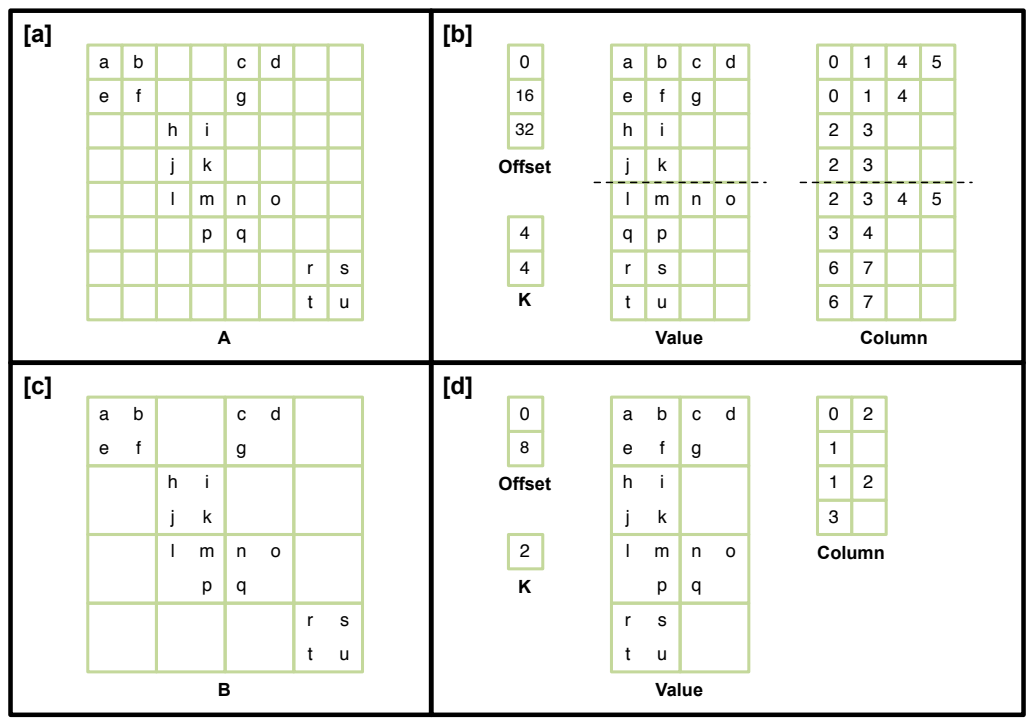

Fig. 5. Sparse matrix [a], WELL [b], sparse blocked matrix [c] and WELL with blocking [d].

In order to guarantee coalesced memory access at warp level, the memory layout of Value [] needs to be redesigned. The overall idea is to transform each $2 \mathrm{D}$ block as a $1 \mathrm{D}$ array $\left(e . g\right.$. $\left[\begin{array}{cc}a & b \\ e & f\end{array}\right]$ becomes $\left.[a, b, e, f]\right)$ and then store those arrays in a interleaved fashion at warp granularity, repeating the process for each batch of nonzeros blocks. Figure 6 clarifies this memory layout with an example. The interleaving placement is highlighted with different shades of gray. The first coalesced memory access will load $[a][h][l][r]$ (one per lane), which is the first element in each block. The second access will load $[b][i][\mathrm{m}][\mathrm{s}]$, and so on until the entire first batch of blocks is completely loaded. This is then repeated for all the other block batches until the warp completes the processing to its local (blocked) ELL structure. Blocking can naturally take advantage of buffering. Moreover, blocking can be integrated with nonzero unrolling (which will now happen at block granularity). For example, if we apply $2 \mathrm{x}$ unrolling to Figure 5, thread lane 0 will buffer two nonzero blocks (i.e. $[a, b, e, f, c, d, g, 0]$ and $[0,2])$ plus 4 elements from $\mathbf{x}$ (i.e. $\left.\left[x_{0}, x_{1}, x_{3}, x_{4}\right]\right)$. The resulting dot product computation can be aggressively optimized with loop unrolling.

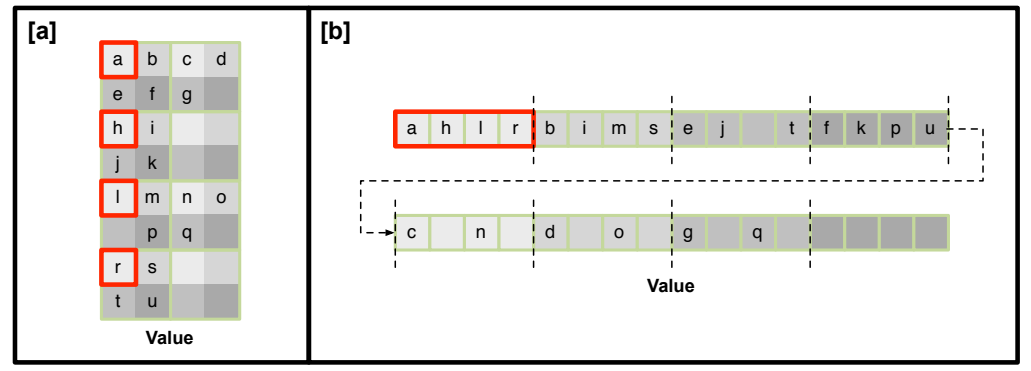

Fig. 6. Sparse blocked matrix [a] and its interleaved memory layout [b].

The blocking technique applied on WELL may be more or less effective, depending on how well an arbitrary blocking factor $[b n \times b m]$ suits the underlying dense substructure in the sparse matrix A. First, we define a $[0,1]$ metric called block density $d_{[b n \times b m]}$ as

$$
d_{[b n \times b m]}=\frac{\mathrm{nnz}}{\mathrm{bn} \cdot \mathrm{bm} \cdot \mathrm{nnz}_{[b n \times b m]}}
$$

where $\mathrm{nnz}_{[b n \times b m]}$ is the number of nonzero blocks in the blocked matrix $\mathbf{B}_{[b n \times b m]}$ extracted from the original 
A. Note that $d_{[b n \times b m]}=1$ when no padding needs to be added. In the example of Figure $5, d_{[2 \times 2]}=0.875$ because we added some zeros to densely fill $\left[\begin{array}{ll}c & d \\ g & 0\end{array}\right],\left[\begin{array}{ll}l & m \\ 0 & p\end{array}\right]$ and $\left[\begin{array}{ll}n & 0 \\ q & 0\end{array}\right]$. The next factor to analyze is the impact of blocking on the WELL data structure. Let $n_{[b n \times b m]}^{w}=\left\lceil\frac{[n / b n]}{w}\right\rceil$ be the number of warps necessary for the blocked matrix $\mathbf{B}_{[b n \times b m]}$ and $k_{[b n \times b m]}^{i}$ be the longest blocked row in the local (blocked) ELL structure associated with warp $i$. Hence, we modify the efficiency formula (6) as follows

$$
e_{[b n \times b m]}=\frac{\mathrm{nnz}_{[b n \times b m]}}{\sum_{i=1}^{n_{b b \times b m]}^{w}} w \cdot k_{[b n \times b m]}^{i}}=\frac{\mathrm{nnz}_{[b n \times b m]}}{\operatorname{Offset}\left[n_{[b n \times b m]}^{w}\right]} .
$$

Considering again the example in Figure 5, $e_{[2 \times 2]}=0.75$ because 2 blocks out 8 have been inserted as zero padding. The last factor to analyze is the effect of blocking on the memory footprint. Given a nonzero block, the amount of data necessary for column indexing will be compressed by a $b n \cdot b m$ factor. Assuming single-precision computation, we define the compression $c^{[b n \times b m]}$ associated with blocking as follows

$$
c_{[b n \times b m]}=\frac{8}{4+\frac{4}{\mathrm{bn} \cdot \mathrm{bm}}} \leq 2,
$$

where the upper bound for compression is 2 (i.e. the scenario in which we represent the sparse matrix with an unique dense block). It might be argued that compression $c_{[b n \times b m]}$ is only dependent by the given blocking factor $[b n \times b m]$ whereas density $d_{[b n \times b m]}$ and efficiency $e_{[b n \times b m]}$ also depend by the original sparse matrix A. We can repeat the same analysis for double-precision computation obtaining

$$
c_{[b n \times b m]}=\frac{12}{8+\frac{4}{\mathrm{bn} \cdot \mathrm{bm}}} \leq 1.5,
$$

where the upper bound is smaller due to the smaller impact of column index compression (and, hence, of blocking) on the overall memory footprint. Finally, we summarize all previous considerations into a quality metric that provides a rough estimate of which blocking factor $[b n \times b m]$ to choose for optimality

$$
q_{[b n \times b m]}=d_{[b n \times b m]} \cdot e_{[b n \times b m]} \cdot c_{[b n \times b m]} .
$$

In other words, $q_{[b n \times b m]}$ estimates the effect of zero padding and compression on the memory footprint associated with a particular choice of $[b n \times b m]$. The definitions of density $d_{[b n \times b m]}$, efficiency $e_{[b n \times b m]}$ and compression $c_{[b n \times b m]}$ are generalization of the base case [1×1] with no blocking. From (11), $q_{[1 \times 1]}=$ $d_{[1 \times 1]} \cdot e_{[1 \times 1]} \cdot c_{[1 \times 1]}=1 \cdot e_{W E L L} \cdot 1=e_{W E L L}$. By comparison, the quality metric $q_{[b n \times b m]}$ may estimate whether applying blocking is a good idea (i.e. presence of a dense substructure) and, eventually, which blocking factor provides a more compact memory footprint (and, likely, good SpMV performance).

\subsection{Empirical evaluation}

Here we analyze the effectiveness of the blocking technique just presented, with the secondary goal to directly correlate the proposed quality metric to measured performance. We considered all the blocking factors with area $b n \cdot b m \in[1,9]$ (e.g. for area $b n \cdot b m=4$ we consider [1 $\times 4],[2 \times 2]$ and [4 $\times 1]$ ). Table 10 reports the SpMV performance on the regular matrices focusing on the baseline $[1 \times 1]$ and the best performing blocking factor. Those results are incremental over those in Section 4, in the sense that the implemented SpMV kernels use both blocking and nonzero unrolling (we report the best-tuned result). As we can visually notice (highlighted and in bold), most of the regular matrices have a dense substructure that can be leveraged with blocking to achieve superior performance. Indeed, we have an average of $1.17 \mathrm{x}$ (1.09x) speedup for single-precision (double-precision). Not surprisingly, the quality metric can estimate fairly well when it is convenient to apply blocking. Specifically, the best performing blocking factor always has a higher quality metric. For example, matrix QCD has a [3 $\times 3]$ dense substructure on which blocking improves performance from 47.215 to 76.215 GFLOPS (single-precision). This improvement can be 


\begin{tabular}{|c|c|c|c|c|c|c|c|c|c|c|}
\hline \multirow[b]{2}{*}{ Matrix } & \multicolumn{3}{|c|}{ Performance [GFLOPS] } & \multicolumn{2}{|c|}{ Quality Metric } & \multicolumn{3}{|c|}{ Performance [GFLOPS] } & \multicolumn{2}{|c|}{ Quality Metric } \\
\hline & [1x1] & \multicolumn{2}{|c|}{ Best } & {$[1 \times 1]$} & Best & [1x1] & \multicolumn{2}{|c|}{ Best } & {$[1 \times 1]$} & Best \\
\hline Circuit & 12.007 & 12.007 & {$[1 \times 1]$} & 0.398 & 0.398 & 9.229 & 9.229 & {$[1 \times 1]$} & 0.398 & 0.398 \\
\hline Dense & 16.163 & 24.180 & [1x9] & 0.992 & 1.780 & 15.526 & 22.350 & [1x9] & 0.992 & 1.405 \\
\hline Economics & 13.333 & 13.333 & {$[1 \times 1]$} & 0.275 & 0.275 & 9.040 & 9.040 & {$[1 \times 1]$} & 0.275 & 0.275 \\
\hline Epidemiology & 38.945 & 38.945 & {$[1 \times 1]$} & 0.999 & 0.999 & 25.991 & 25.991 & {$[1 \times 1]$} & 0.999 & 0.999 \\
\hline FEM/Accelerator & 38.271 & 40.032 & {$[2 \times 1]$} & 0.840 & 0.861 & 25.572 & 25.572 & [1x1] & 0.840 & 0.840 \\
\hline FEM/Cantilever & 44.253 & 68.577 & {$[3 \times 3]$} & 0.881 & 1.435 & 30.475 & 37.330 & [3x3] & 0.881 & 1.133 \\
\hline FEM/Harbor & 31.382 & 37.155 & [1x3] & 0.663 & 0.792 & 21.989 & 23.444 & [1x2] & 0.663 & 0.705 \\
\hline FEM/Ship & 44.159 & 72.493 & {$[1 \times 6]$} & 0.846 & 1.450 & 29.728 & 40.788 & [1x6] & 0.846 & 1.172 \\
\hline FEM/Spheres & 48.005 & 79.597 & {$[3 \times 3]$} & 0.930 & 1.635 & 32.555 & 42.509 & {$[3 \times 3]$} & 0.930 & 1.290 \\
\hline Ga41As41H72 & 19.268 & 20.523 & [1x2] & 0.364 & 0.390 & 12.898 & 12.898 & {$[1 \times 1]$} & 0.364 & 0.364 \\
\hline Protein & 40.783 & 59.862 & {$[3 \times 3]$} & 0.796 & 1.325 & 27.391 & 34.863 & [1x3] & 0.796 & 1.024 \\
\hline QCD & 47.929 & 76.215 & [3x3] & 1.000 & 1.800 & 32.661 & 43.447 & [3x3] & 1.000 & 1.421 \\
\hline Si41Ge41H72 & 21.406 & 22.700 & [1x2] & 0.410 & 0.441 & 14.341 & 14.341 & {$[1 \times 1]$} & 0.410 & 0.410 \\
\hline Wind Tunnel & 48.713 & 64.080 & [1x3] & 0.982 & 1.326 & 33.254 & 37.955 & [1x3] & 0.982 & 1.137 \\
\hline \multirow[t]{2}{*}{ Harmonic Mean } & 26.196 & $1.17 x$ & & & & 18.715 & $1.09 x$ & & & \\
\hline & \multicolumn{5}{|c|}{ Single Precision } & \multicolumn{5}{|c|}{ Double Precision } \\
\hline
\end{tabular}

Table 10. Incremental performance for the blocking technique on the regular benchmarks.

correlated to the quality metric improvement from 1.000 to 1.800 . We analyzed the relationship between the measured performance and the quality metric across all the blocking factors more directly. There is a positive correlation of 0.910 (single-precision) and 0.942 (double-precision). For the sake of completeness, we also report performance tests on irregular matrices (Table 11). Even here, despite comparing with a poor baseline (we have not yet addressed the irregularity issue), we can observe that the blocking technique provides a performance edge that we can integrate with other advanced techniques for SpMV optimization. On the other hand, the skewed distribution of some of the irregular matrices makes the proposed quality metric less reliable, although the correlation with performance still remains 0.796 (single-precision) and 0.885 (double-precision).

\begin{tabular}{|c|c|c|c|c|c|c|c|c|c|c|}
\hline \multirow{3}{*}{$\begin{array}{c}\text { Matrix } \\
\text { Circuit5M }\end{array}$} & \multicolumn{3}{|c|}{ Performance [GFLOPS] } & \multicolumn{2}{|c|}{ Quality Metric } & \multicolumn{3}{|c|}{ Performance [GFLOPS] } & \multicolumn{2}{|c|}{ Quality Metric } \\
\hline & {$[1 \times 1]$} & \multicolumn{2}{|c|}{ Best } & {$[1 \times 1]$} & Best & {$[1 \times 1]$} & \multicolumn{2}{|c|}{ Best } & {$[1 \times 1]$} & Best \\
\hline & 0.393 & 0.589 & [1x9] & 0.247 & 0.214 & 0.383 & 0.517 & [1x4] & 0.247 & 0.237 \\
\hline Eu-2005 & 13.094 & 14.286 & [11x2] & 0.457 & 0.438 & 10.641 & 10.641 & {$[1 \times 1]$} & 0.457 & 0.457 \\
\hline In-2004 & 11.988 & 15.208 & [1x3] & 0.367 & 0.367 & 10.223 & 10.870 & {$[1 \times 2]$} & 0.367 & 0.346 \\
\hline LP & 1.643 & 1.964 & {$[1 \times 2]$} & 0.220 & 0.231 & 1.568 & 1.568 & {$[1 \times 1]$} & 0.220 & 0.220 \\
\hline Mip1 & 1.307 & 2.000 & [1x9] & 0.698 & 1.039 & 1.282 & 1.909 & [1x9] & 0.698 & 0.820 \\
\hline Webbase & 4.880 & 4.880 & {$[1 \times 1]$} & 0.316 & 0.316 & 4.529 & 4.529 & {$[1 \times 1]$} & 0.316 & 0.316 \\
\hline \multirow[t]{2}{*}{ Harmonic Mean } & 1.401 & $1.40 x$ & & & & 1.351 & $1.28 x$ & & & \\
\hline & \multicolumn{5}{|c|}{ Single Precision } & \multicolumn{5}{|c|}{ Double Precision } \\
\hline
\end{tabular}

Table 11. Incremental performance for the blocking technique on the irregular benchmarks.

\section{Loseless compression for indexing data}

In general, compressing the sparse matrix structure (i.e indexing data) is a target for optimization since it reduces the memory footprint. For example, the blocking technique just analyzed substantially reduces the column indices associated with each nonzero block, providing a $[b n \times b m]$ implicit compression ratio. There are additional opportunities for compression within the nonzero pattern. However, any technique that we may want to adopt should have a decompression phase which is as simple as possible in order to be efficiently embedded into the SpMV kernel. Following this principle, we proposed a technique based on delta encoding of column indices [31]. Let us consider the way thread lanes process nonzeros within a 


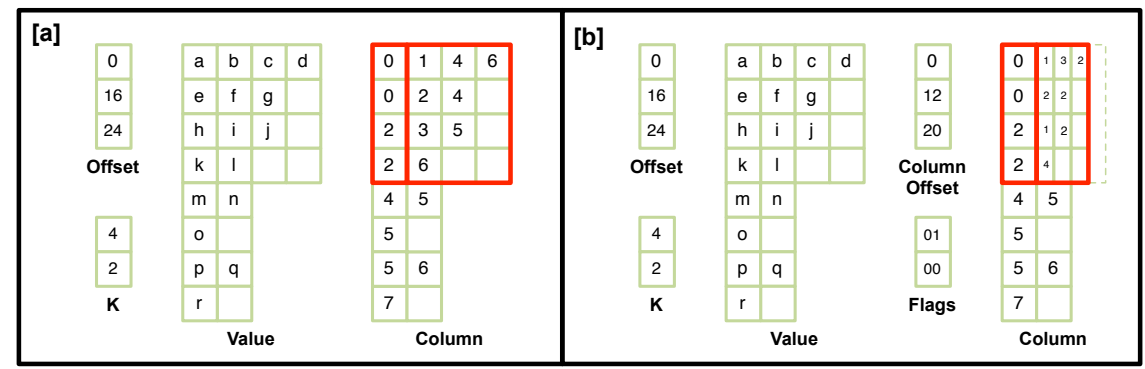

Fig. 7. WELL [a] and WELL with delta-based index compression [b]. In this particular example, the column indices in the first warp are close enough to apply differential encoding and reduce the associated memory footprint.

warp. Whenever the column distance between two consecutive elements is small enough, it is convenient to reconstruct the next column using a delta from the current index. This, in turn, allows to simply store a 8-bit or 16-bit delta rather than a 32-bit absolute index. This compression technique can be tailored to SIMD vectorized execution. Specifically, we propose to apply the differential encoding only when all the delta within the warp (i.e. local ELL data structure) are representable as 8-bit or 16-bit integers. This provides a regular memory layout as well as a divergency free execution. Figure 7 shows an example of our compression technique integrated into a sparse matrix format with warp granularity. The nonzero structure associated with the first warp is stored as an absolute base column index (from the first nonzero) followed by a sequence of compressed delta values occupying in the case of the example half the space (of 32-bit integers). The correspondent portion of Column [] is now resized down from dimension 4 to 2.5. However, we may decide to introduce some padding in order to $w$-align the next warp (i.e. moving the second warp from location 10 to 12). This delta-based compression technique needs some additional data structures. First, it is necessary to store ColumnOffset[], an incremental offset to locate any local indexing data in Column []. Second, we need a flag array Flags [] that encodes the compression applied to each warp (e.g. $b_{1} b_{0}=00$ is "no compression", $b_{1} b_{0}=01$ is " 16 -bit encoding" and $b_{1} b_{0}=10$ is "8-bit encoding"). The second warp in Figure 7 is not compressed despite being eligible. In general, it is not profitable to apply delta encoding to warps with small $k_{i}=\{1,2\}$ because they do not improve the local memory footprint after the necessary padding for $w$-alignment. Finally, this compression technique can be easily integrated with blocking due to the independency of column indexing data. Similarly, it is possible to apply nonzero unrolling by buffering deltas rather than absolute column indices.

We can now revise the quality metric $q_{[b n \times b m]}$ defined in Section 5 in order to incorporate our delta-based compression technique. Given an arbitrary warp $w^{[i]}$, we can define its delta compression as

$$
\delta_{[b n \times b m]}^{i}=\left\{\begin{array}{cl}
1 & \text { no delta encoding } \\
\frac{4 k_{i}}{\left.\left.4+\left.4 w\right|_{[b n \times b m]} ^{i}-1\right) / 2 w\right\rceil} \leq 2 & \text { if 16-bit delta encoding } \\
\frac{4 k_{i}}{\left.\left.4+\left.4 w\right|_{[b n \times b m]} ^{i}-1\right) / 4 w\right\rceil} \leq 4 & \text { if 8-bit delta encoding }
\end{array} .\right.
$$

Here the achieved ratio has a different upper bound, depending on which compression it is possible to apply. This definition takes into account $w$-alignment. Specifically, the ceiling function at the denominator evaluates the number of aligned memory chunks associated with differential encoding. Note also how the delta compression $d c_{[b n \times b m]}^{i}$ depends on the blocking factor $[b n \times b m]$. For example, doubling the block dimension $b m$ halves all the column deltas of the associated blocked matrix $\mathbf{B}$. As a result, some warp may 
become eligible for compression. The overall delta compression $d c_{[b n \times b m]}$ is defined as follows

$$
\delta_{[b n \times b m]}=\frac{\sum_{i=1}^{n_{[b n \times b m]}^{(w)}} k_{[b n \times b m]}^{i} \cdot d c_{[b n \times b m]}^{i}}{\sum_{i=1}^{i} k_{[b n \times b m]}^{i} n^{[b n \times b m]}}=\frac{\operatorname{ColumnOffset}\left[n_{[b n \times b m]}^{w}\right]}{\operatorname{Offset}\left[n_{[b n \times b m]}^{w}\right]} .
$$

The weighted average across the warps can be quickly obtained by dividing the size of Value [] by the size of Column[]. Referring to the example in Figure 7, $d c_{[b n \times b m]}=24 / 20=1.2$. Last, we update definition (9) and (10) in order to integrate the newly defined delta compression $d c_{[b n \times b m]}$

$$
\begin{gathered}
c_{[b n \times b m]}=\frac{8}{4+\frac{4}{\mathrm{bn} \cdot \mathrm{bm} \cdot \delta_{[b n \times b m]}}} \leq 2, \\
c_{[b n \times b m]}=\frac{12}{8+\frac{4}{\mathrm{bn} \cdot \mathrm{bm} \cdot \delta_{[b n \times b m]}}} \leq 1.5 .
\end{gathered}
$$

Intuitively, the ability to apply delta compression to a sparse matrix increases $c_{[b n \times b m]}$ and, hence, the quality metric $q_{[b n \times b m]}$.

\subsection{Empirical evaluation}

We performed some computational experiments with the aim to evaluate the incremental benefit of deltabased index compression over the other optimization techniques. Table 12 reports the best-tuned (in terms of blocking and unrolling factors) SpMV performance with and without delta-compression on the regular benchmark suite. In general, we observed an average of 1.09x (1.05x) speedup factor for single-precision (double-precision) SpMV computation over a fairly optimized baseline. As we can see (highlighted and in bold), almost all the matrices have a nonzero pattern that can be successfully exploited to reduce memory footprint and to improve SpMV performance. The only exception is the matrix Dense. As mentioned, the row-based parallelization implemented by the underlying WELL does not generate a sufficient number of warps to properly exploit the GPU hardware architecture for that matrix. Therefore, we can treat that matrix as an outlier. Moreover, the revised quality metric is now able to capture the memory footprint reduction associated with the compression technique. In other words, a higher value of $q_{[b n \times b m]}$ correlates with superior performance.

Table 13 reports the result for the other set of matrices. Observing the negligible increments of the quality metric, we can deduce that there is no real opportunity to leverage the irregular nonzero patterns to improve the memory footprint. As a consequence, there is no substantial effect on the average SpMV performance. On the other hand, we can observe that the additional complexity of integrating the compression technique into a SpMV kernel does not introduce any visible performance overhead. This provides a supporting evidence to always apply the proposed optimization, without the need of introducing an additional variable to the space of tuning parameters.

In the last sections, we presented two complementary optimization techniques explicitly targeted to reducing memory footprint. We could observe tangible performance improvements, especially for matrices with regular structure. We now switch our focus on coping with matrix irregularity.

\section{Improving Adaptive ELL}

The ability to adapt the nonzero workload of each threads (as opposed to row-based parallelization used so far) provides an effective way to cope with matrix irregularity. We proposed AdELL [25], a warp-grained 


\begin{tabular}{|c|c|c|c|c|c|c|c|c|}
\hline \multirow[b]{3}{*}{ Matrix } & \multicolumn{2}{|c|}{ [GFLOPS] } & \multicolumn{2}{|c|}{ Quality Metric } & \multicolumn{2}{|c|}{ [GFLOPS] } & \multicolumn{2}{|c|}{ Quality Metric } \\
\hline & \multicolumn{4}{|c|}{ Delta Compression } & \multicolumn{4}{|c|}{ Delta Compression } \\
\hline & w/o & $\mathrm{w} /$ & w/o & $\mathrm{w} /$ & w/o & $\mathrm{w} /$ & w/o & $\mathrm{w} /$ \\
\hline Circuit & 12.007 & 12.326 & 0.398 & 0.419 & 9.229 & 9.445 & 0.398 & 0.412 \\
\hline Dense & 24.180 & 23.584 & 1.780 & 1.924 & 22.350 & 21.669 & 1.405 & 1.463 \\
\hline Economics & 13.333 & 16.089 & 0.275 & 0.356 & 9.040 & 10.235 & 0.275 & 0.324 \\
\hline Epidemiology & 38.945 & 39.361 & 0.999 & 1.152 & 25.991 & 26.060 & 0.999 & 1.096 \\
\hline FEM/Accelerator & 40.032 & 42.114 & 0.861 & 0.972 & 25.572 & 27.789 & 0.840 & 0.924 \\
\hline FEM/Cantilever & 68.577 & 71.351 & 1.435 & $\mathbf{1 . 5 1 0}$ & 37.330 & 38.978 & 1.133 & 1.187 \\
\hline FEM/Harbor & 37.155 & 41.839 & 0.792 & 0.958 & 23.444 & 25.238 & 0.705 & 0.791 \\
\hline FEM/Ship & 72.493 & 74.980 & 1.450 & 1.551 & 40.788 & 41.088 & 1.172 & 1.214 \\
\hline FEM/Spheres & 79.597 & 81.399 & 1.635 & 1.717 & 42.509 & 43.934 & 1.290 & 1.274 \\
\hline Ga41As41H72 & 20.523 & 24.140 & 0.390 & 0.484 & 12.898 & 15.060 & 0.364 & 0.436 \\
\hline Protein & 59.862 & 62.970 & 1.325 & 1.368 & 34.863 & 37.111 & 1.024 & 1.104 \\
\hline QCD & 76.215 & 78.990 & 1.800 & 1.887 & 43.447 & 43.910 & 1.421 & 1.456 \\
\hline Si41Ge41H72 & 22.700 & 26.485 & 0.441 & 0.528 & 14.341 & 16.774 & 0.410 & 0.491 \\
\hline Wind Tunnel & 64.080 & 73.236 & 1.326 & 1.588 & 37.955 & 41.143 & 1.137 & 1.254 \\
\hline \multirow[t]{2}{*}{ Harmonic Mean } & 30.675 & $1.09 x$ & & & 20.465 & $1.04 x$ & & \\
\hline & \multicolumn{4}{|c|}{ Single Precision } & \multicolumn{4}{|c|}{ Double Precision } \\
\hline
\end{tabular}

Table 12. Incremental performance for delta compression on the regular benchmarks.

\begin{tabular}{|c|c|c|c|c|c|c|c|c|}
\hline \multirow[b]{3}{*}{ Matrix } & \multicolumn{2}{|c|}{ [GFLOPS] } & \multicolumn{2}{|c|}{ Quality Metric } & \multicolumn{2}{|c|}{ [GFLOPS] } & \multicolumn{2}{|c|}{ Quality Metric } \\
\hline & \multicolumn{4}{|c|}{ Delta Compression } & \multicolumn{4}{|c|}{ Delta Compression } \\
\hline & $\mathrm{w} / \mathrm{o}$ & $\mathrm{w} /$ & $\mathrm{w} / \mathrm{o}$ & $\mathrm{w} /$ & $\mathrm{w} / \mathrm{o}$ & $\mathrm{w} /$ & $\mathrm{w} / \mathrm{o}$ & $\mathrm{w} /$ \\
\hline Circuit5M & 0.589 & 0.579 & 0.214 & 0.228 & 0.517 & 0.506 & 0.237 & 0.238 \\
\hline Eu-2005 & 14.286 & 14.302 & 0.438 & 0.458 & 10.641 & 10.856 & 0.457 & 0.476 \\
\hline In-2004 & 15.208 & 15.647 & 0.367 & 0.409 & 10.870 & 11.261 & 0.346 & 0.377 \\
\hline LP & 1.964 & 1.963 & 0.231 & 0.231 & 1.568 & 1.783 & 0.208 & 0.208 \\
\hline Mip1 & 2.000 & 1.916 & 1.039 & 1.091 & 1.909 & 1.787 & 0.820 & 0.841 \\
\hline Webbase & 4.880 & 4.925 & 0.316 & 0.326 & 4.529 & 4.553 & 0.316 & 0.323 \\
\hline \multirow[t]{2}{*}{ Harmonic Mean } & 1.969 & $0.99 x$ & & & 1.713 & $1.00 x$ & & \\
\hline & \multicolumn{4}{|c|}{ Single Precision } & \multicolumn{4}{|c|}{ Double Precision } \\
\hline
\end{tabular}

Table 13. Incremental performance for delta compression on the irregular benchmarks.

sparse matrix format implementing the adaptivity technique. AdELL is based on the idea of allocating an adaptive number of warp lanes $l_{r}$ to each row $r$, depending on the row's nonzeros $n n z_{r}$. In other words, now each warp $w^{[i]}$ provides a more flexible computation strategy where an arbitrary number $\{1,2, \ldots, w\}$ of rows can be used to keep all the processing lanes occupied (i.e. $\sum_{r \in w^{[i]}} l_{r}=w$ ). In general, thread lanes need to collaborate to aggregate the dot product of the corresponded row $r$. This can be efficiently implemented as a warp-level segmented reduction by building upon intrinsics (e.g. warp shuffle instruction). A careful lane assignment can greatly improve the overall memory footprint efficiency (which is still calculated as $e^{W E L L}$ ). Specifically, each warp workload $k_{i}$ is now determined by value of the largest $\left\lceil\frac{n n z_{r}}{t_{r}}\right\rceil$ among the rows (rather than the absolute $n n z_{r}$ ). Irregular rows (i.e. with a large number of nonzeros) can now be processed by up to $w$ lanes or even across multiple warps by the means of atomic operations, providing an effective strategy to implement load balancing.

The example in Figure 8 illustrates the AdELL data structure and its efficiency benefit over WELL. A reduction map array Map [] is used to specify each local segmented reduction pattern (e.g. warp $w^{[0]}$ has Map [0] $=1001$ since $l_{0}=3$ and $l_{1}=1$ ). Moreover, an additional array RowOffset [] is used to store the first row associated with each warp. This is necessary because the implicit assignment of $w$ rows for each warp does not hold anymore. AdELL has the same fundamental warp-grained structure of WELL so all the presented optimization techniques can be directly applied. Considering blocking, the adaptivity technique will just focus on allocating lanes to rows from the blocked matrix. Similarly, delta-based compression can 


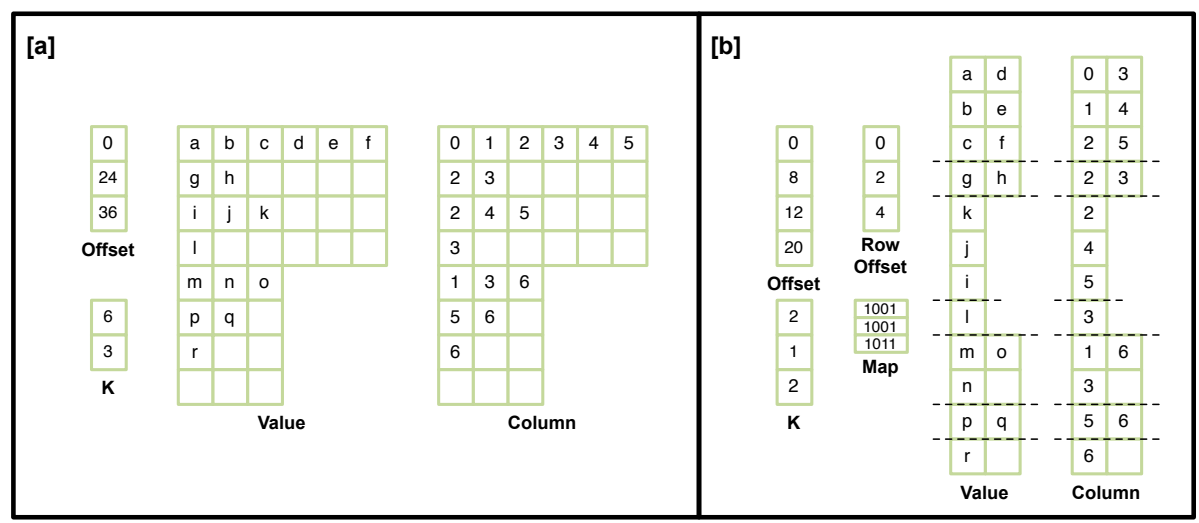

Fig. 8. An example of the nonzeros distribution unfavorable to row-based parallelization. On the one hand, WELL [a] needs two warps to process 7 rows and introduces a substantial amount of zero padding. On the other hand, AdELL can assign an arbitrary number of lanes to each row (e.g. row 0 has $l_{0}=3$ lanes) obtaining an evident efficiency improvement $\left(e^{W E L L}\right.$ increases from $18 / 36=0.5$ to $18 / 20=0.9)$. Indeed, AdELL makes effective use of a total of 12 lanes over 3 warps

be applied on Column[] and each lane can unroll the processing of its nonzero blocks. Figure 8 does not show any example of rows distributed across multiple warp. In that case, we may use an addition flag (e.g. $b_{2}$ in Flags []) to signal that the final step of segmented reduction is an atomic operation on result vector $\mathbf{y}$.

AdELL is a data structure that supports adaptivity, a technique can be leveraged to optimize the SpMV performance for irregular matrices. However, it is the actual warp assignment that achieves memory footprint efficiency and load-balancing across the GPU hardware architecture. We can define a simplified version of this Warp-Balancing Problem [25]. Given the set of rows from (blocked) matrix A, we perform the following (constrained) tasks :

Lane Assignment : Assign an adaptive number of lanes $l_{r} \in\{1,2, \ldots, w\}$ to each row $r$

Warp Partitioning : Compose each warp $w^{[i]}$ such that $\sum_{r \in w^{[i]}} l_{r}=w$

The multi-objective function maximizes the memory footprint efficiency $e^{W E L L}$ while minimizing the global workload unbalance $u=\frac{\max k_{i}-k_{\text {median }}}{k_{\text {median }}}$ across the warps. Here, we do not combine the two objectives into one (e.g. linear combination) since it is more appropriate to generalize rather than focus on a specific formulation. The optimization problem is then to find a lane assignment and a partition that optimize the given multi-objective function. Note, the problem just stated is in the class of NP-hard load-balancing problems [42]. We focus on a variation where rows can be distributed across multiple warps and where the original row ordering should be maintained. Here we propose a revised heuristic to efficiently solve the Warp-Balancing Problem and, consequently, improve the SpMV performance. This $O(n)$-runtime approach is designed to be faster than the original self-balancing heuristic used by AdELL [25], with the aim to reduce the cost of preprocessing. Our revised heuristic, in turns, achieves both efficient lane assignment (with Algorithm 1) and warp partitioning (with Heuristic 2).

Let first describe a lane assignment approach to compose candidate warps with minimal $k_{i}$ and, thus, with maximal $e^{E L L}$. Given a set of $r \leq w$ consecutive rows $\{1, . ., r\}$, we solve the subproblem of assigning warp lanes to the rows so that the resulting warp $w^{[i]}$ has minimal $k_{i}$ and, thus, maximal $e_{i}^{E L L}$. The greedy approach presented by Algorithm 1 is incremental. In other words, we use the optimal warp lane assignment for $\{1, . ., r-1\}$ in order to construct the one for $\{1,2, . ., r-1, r\}$. Given an optimal warp $w^{[i]}$ with minimal $k_{i}$, we calculate the lanes $l_{r}$ potentially available for row $r$ (lines 1 to 5 ). $l_{r}$ may be sufficient to process $\mathrm{nnz}_{r}$ nonzeros. Otherwise, we have to increase $k_{i}$ by removing one of the lanes assigned to the rows (lines 9 to 10). If no removal is possible (i.e. all rows have a single lane), we update $k_{i}$ only considering how to accommodate $\mathrm{nnz}_{r}$ nonzeros over $l_{r}$ lanes (line 12). Finally, we can add the new row $r$ (line 16).

We can prove the optimality of this approach by induction on the rows incrementally added to the warp. The base case is an empty warp $w^{[i]}$ with $k_{i}=0$. In that case, $k_{i}$ is updated to the minimal $\left\lceil\frac{\mathrm{nnz}_{r}}{w}\right\rceil$ to 


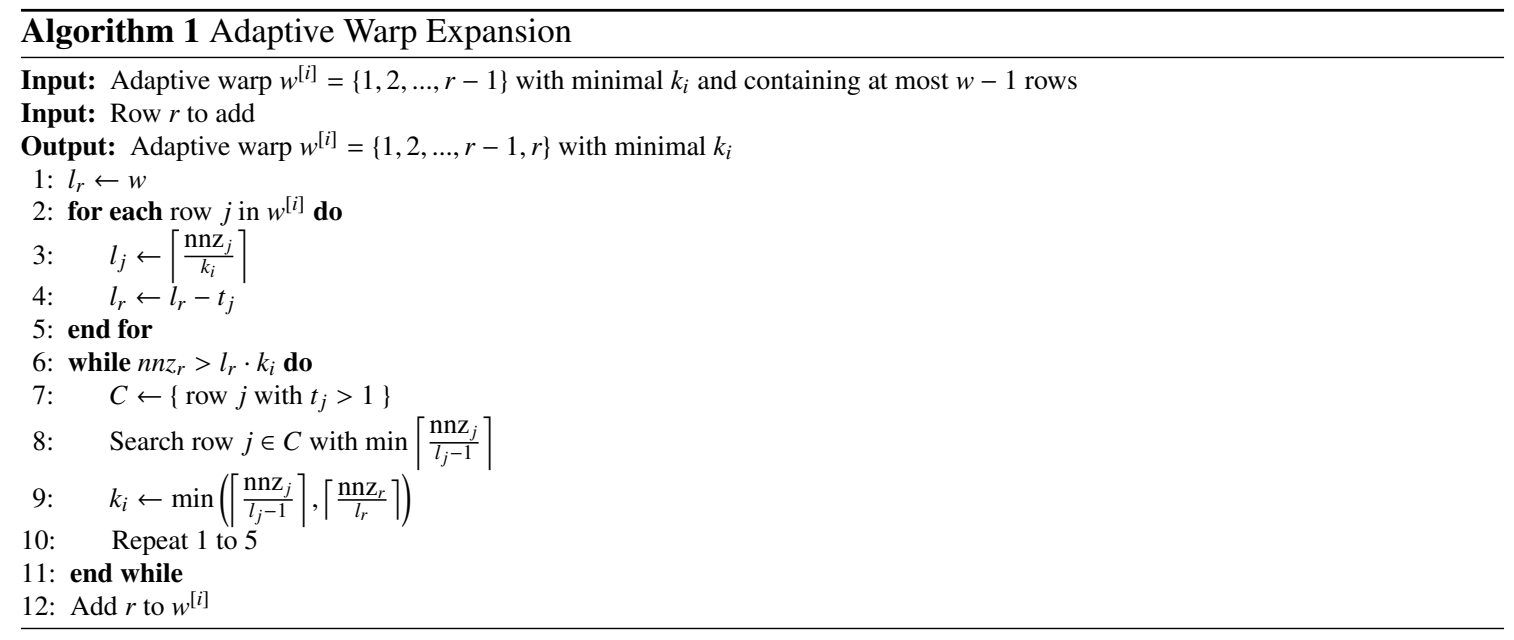

accommodate $\mathrm{nnz}_{r}$ nonzeros. The induction step can be proved by focusing on the lane assignment. When $l_{r} \cdot k_{i}^{r-1}$ is sufficient to process $\mathrm{nnz}_{r}$, we just keep $k_{i}^{r}=k_{i}^{r-1}$. Otherwise, we need to increase $k_{i}^{r-1}$ either to make the current $l_{r}$ lanes "longer" (i.e. capable of processing more nonzeros) or to move the assignment of one or more lanes from row $j \in\{1,2, . ., r-1\}$ to row $r$ (i.e. increasing $l_{r}$ ). Note, the assignment between each row $j$ and its $l_{j}$ lanes follows the modulo arithmetic. Whenever we increase $k_{i}$ by one, each row $j$ may have either $l_{j}=\left\lceil\frac{\mathrm{nnz}_{j}}{k_{i}+1}\right\rceil=\left\lceil\frac{\mathrm{nnz}_{j}}{k_{i}}\right\rceil$ (same assignment) or $l_{j}=\left\lceil\frac{\mathrm{nnz}_{j}}{k_{i}+1}\right\rceil<\left\lceil\frac{\mathrm{nnz}_{j}}{k_{i}}\right\rceil$ (reduced number of lanes). On the other hand, the quantity $l_{r} \cdot k_{i}$ is monotonically increasing (recall that $l_{r}=w-\sum_{j=1}^{r-1} l_{j}$ ). Given $k_{i}^{r-1}$, we can find the optimal $k_{i}^{*}$ by unit increments over $k_{i}^{r-1}$ until $k_{i} \cdot l_{r} \geq n n z_{r}$. It might be argued that Algorithm 1 goes from the optimal $k_{i}^{r-1}$ for rows $\{1,2, \ldots, r-1\}$ to $k_{i}^{r-1}$ for rows $\{1,2, \ldots, r-1, r\}$ with steps that are equivalent to multiple unit increments done at once. Whenever we search for a row $j$ with minimum $k_{i}^{\prime}=\left\lceil\frac{\mathrm{nnz}_{j}}{l_{j}-1}\right\rceil$ (line 8), we aim to move an additional lane from row $j$ to row $r$. Due to the modulo arithmetic, all the intermediate unit increments between $k_{i}$ and $k_{i}^{\prime}$ do not increase $l_{r}$. In other words, only the last step can modify the overall lane assignment and lead to the optimal $k_{i}^{*}$. This reasoning also applies for the case in which it is sufficient expand $k_{i}$ without increasing $l_{r}$ (line 9), proving the optimality of the presented greedy approach.

The warp-balancing heuristic itself is described by Heuristic 2. As we can see, we have two additional parameters $k_{\min }$ and $k_{\max }$ that are available to tune the way AdELL is composed. Specifically, $k_{\min }$ gives a suggestion to favor warps with $k_{i} \geq k_{\min }$. This is motivated by the empirical fact that warps below $k_{\min }=4$ do not optimize the execution efficiency on the underlying GPU architecture. On the other hand, $k_{\max }$ imposes an upper bound on $k_{i}$ that can be used to distribute rows across multiple warps, providing a tuning factor for load-balancing. The proposed heuristic incrementally processes the rows in matrix $(A)$. At each step, it generates a set of $w$ optimal candidate warps using Algorithm 1 (line 3). Then, it greedily selects the best $w^{[i]}$ in terms of efficiency such that $k_{\min } \leq k_{i} \leq k_{\max }$ (line 4 to 11). If this latter condition does not hold for any candidate, we also consider warps satisfying $k_{i}>k_{\max }$. Whenever this happen, the upper limit $k_{\max }$ is subsequently enforced by splitting $w^{[i]}$ on multiple atomic warps with $\left[\frac{k_{i}}{k_{\max }}\right\rceil$ workload (lines 12 to 16). The last part of the heuristic (lines 20 to 22) takes care of the case in which the number of warps $n_{w}$ is too low to fully utilize the GPU hardware (e.g. matrix Dense with WELL). The proposed heuristic clearly has $O(n)$ runtime. In fact, the outer loop over the rows performs constant work (incremental construction of $w$ candidate warps) at each iteration.

\subsection{Empirical evaluation}

The main target of adaptivity is the irregular benchmark suite. As done for the other optimization techniques, we performed some computational tests with the aim to evaluate the incremental benefit in terms of SpMV performance. We composed the AdELL data structure considering all the following upper limits: 


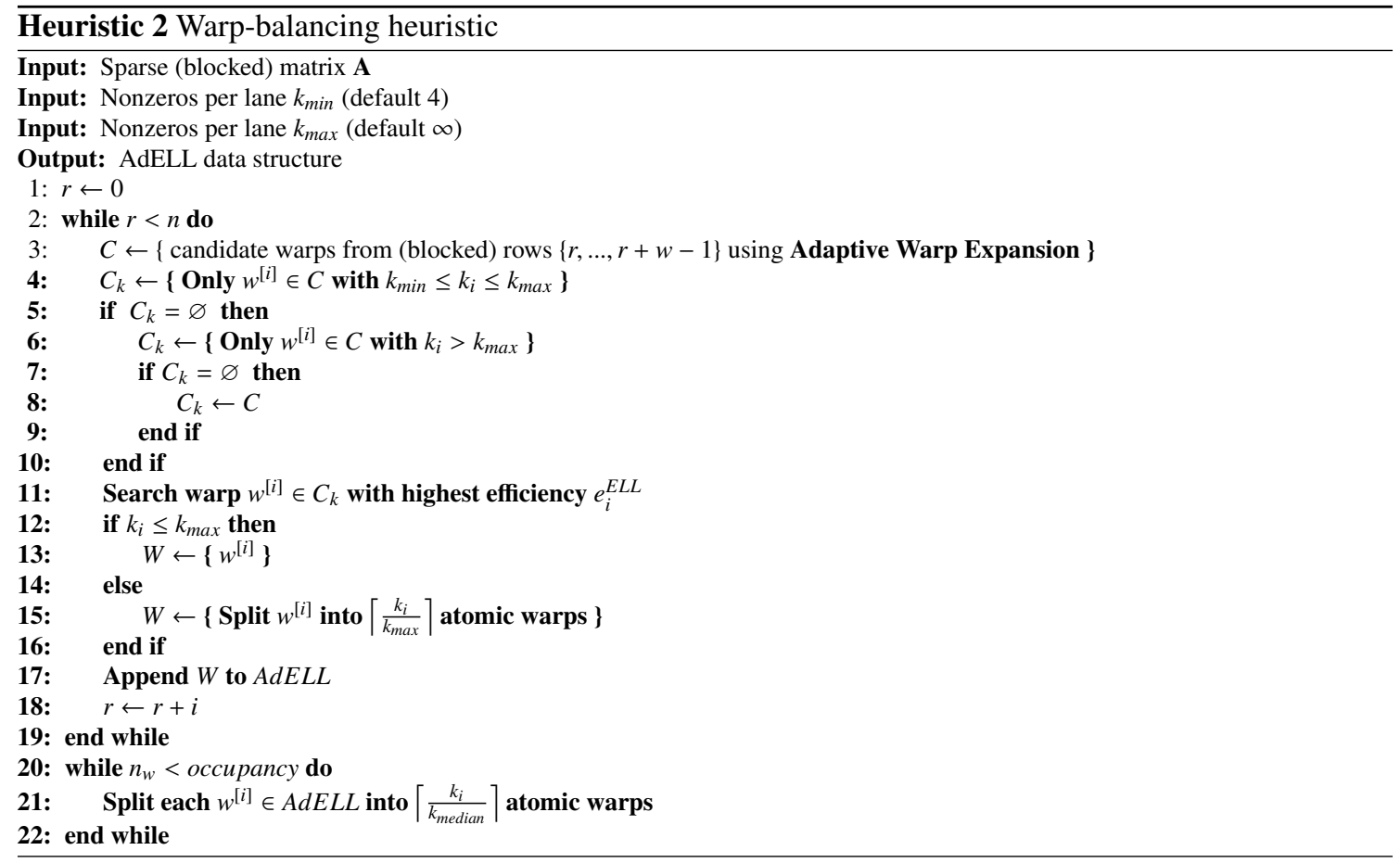

$k_{\max }=\{4,8,16,32,64,128, \infty\}$ (where $\infty$ corresponds to not providing any $k_{\max }$ ). Table 14 reports the best-tuned (in terms of blocking, unrolling factors, upper limit $k_{\max }$ ) SpMV performance with and without adaptivity on the set of irregular matrices. From now on, we will use the term AdELL+ to refer to AdELL with all the optimizations active. Not surprisingly, addressing the irregular matrix structure brings a huge performance improvement on all the benchmark suite (highlighted and in bold). We observe an astonishing 19.93x (14.03x) speedup for single-precision (double-precision) SpMV computation. The simultaneous increment of quality metric $q_{\left[b n \times b m, k_{\max }\right]}$ (notice that we added $k_{\max }$ to the notation) can give us a hint of the effect of adaptivity. The warps selected by the warp-balancing heuristic have by construction a good efficiency $e^{E L L}$ that contributes to improving the overall memory footprint. On the other hand, the imposed upper limit $k_{\max }$ is fundamental for load-balancing. Let us take matrix Circuit5M as example. The best-tuned result is obtained when $k_{\max }=16$. This upper limit generates an AdELL+ data structure with 25233 atomic warps out of 216974. This corresponds to a nearly perfect load-balanced execution where heavyweight rows (accounting for 13\% of the total nonzeros) are broken down into small pieces of computation. Adaptivity is also useful for regular matrices as shown by Table 14. We observed another substantial 1.57x (1.52x)

\begin{tabular}{|c|c|c|c|c|c|c|c|c|}
\hline \multirow[b]{3}{*}{ Matrix } & \multicolumn{2}{|c|}{ [GFLOPS] } & \multirow{2}{*}{\multicolumn{2}{|c|}{ Quality Metric }} & \multicolumn{2}{|c|}{ [GFLOPS] } & \multicolumn{2}{|c|}{ Ouality Metric } \\
\hline & \multicolumn{3}{|c|}{ Adaptivity } & & \multicolumn{4}{|c|}{ Adaptivity } \\
\hline & $\mathrm{w} / \mathrm{o}$ & $\mathrm{w} /$ & $\mathrm{w} / \mathrm{o}$ & $\mathrm{w} /$ & $\mathrm{w} / \mathrm{o}$ & $\mathrm{w} /$ & $\mathrm{w} / \mathrm{o}$ & $\mathrm{w} /$ \\
\hline Circuit5M & 0.589 & 35.870 & 0.214 & 0.952 & 0.517 & 24.669 & 0.237 & 0.985 \\
\hline Eu-2005 & 14.286 & 46.140 & 0.438 & 0.978 & 10.641 & 28.493 & 0.457 & 0.951 \\
\hline In-2004 & 15.208 & 48.664 & 0.367 & 1.095 & 10.870 & 28.677 & 0.346 & 0.936 \\
\hline LP & 1.964 & 27.059 & 0.231 & 1.076 & 1.568 & 17.093 & 0.208 & 0.967 \\
\hline Mip1 & 2.000 & 76.219 & 1.039 & 1.560 & 1.909 & 44.675 & 0.820 & 1.266 \\
\hline Webbase & 4.880 & 28.448 & 0.316 & 0.753 & 4.529 & 17.267 & 0.316 & 0.805 \\
\hline \multirow[t]{2}{*}{ Harmonic Mean } & 1.938 & $\overline{19.93 x}$ & & & 1.716 & $\overline{14.03 x}$ & & \\
\hline & \multicolumn{4}{|c|}{ Single Precision } & \multicolumn{4}{|c|}{ Double Precision } \\
\hline
\end{tabular}

Table 14. Incremental performance for the adaptivity technique on the irregular benchmarks. 


\begin{tabular}{|c|c|c|c|c|c|c|c|c|}
\hline \multirow[b]{3}{*}{ Matrix } & \multicolumn{2}{|c|}{ [GFLOPS] } & \multicolumn{2}{|c|}{ Quality Metric } & \multicolumn{2}{|c|}{ [GFLOPS] } & \multicolumn{2}{|c|}{ Quality Metric } \\
\hline & \multicolumn{4}{|c|}{ Adaptivity } & \multicolumn{4}{|c|}{ Adaptivity } \\
\hline & w/o & $\mathrm{w} /$ & w/o & $\mathrm{w} /$ & w/o & $\mathrm{w} /$ & $\mathrm{w} / \mathrm{O}$ & $\mathrm{w} /$ \\
\hline Circuit & 12.326 & 26.554 & 0.419 & 0.864 & 9.445 & 18.811 & 0.412 & 0.855 \\
\hline Dense & 23.584 & 91.595 & 1.924 & 1.938 & 21.669 & 50.641 & 1.463 & 1.475 \\
\hline Economics & 16.089 & 26.899 & 0.356 & 0.934 & 10.235 & 19.746 & 0.324 & 0.888 \\
\hline Epidemiology & 39.361 & 34.082 & 1.152 & 0.891 & 26.060 & 24.356 & 1.096 & 1.095 \\
\hline FEM/Accelerator & 42.114 & 45.015 & 0.972 & 1.090 & 27.789 & 27.629 & 0.924 & 0.950 \\
\hline FEM/Cantilever & 71.351 & 80.569 & 1.510 & 1.748 & 38.978 & 44.383 & 1.187 & 1.336 \\
\hline FEM/Harbor & 41.839 & 54.889 & 0.958 & 1.301 & 25.238 & 34.045 & 0.791 & 1.092 \\
\hline FEM/Ship & 74.980 & 84.339 & 1.551 & 1.775 & 41.088 & $\mathbf{4 7 . 4 1 0}$ & 1.214 & 1.379 \\
\hline FEM/Spheres & 81.399 & 81.361 & 1.717 & 1.758 & 43.934 & 45.715 & 1.274 & 1.357 \\
\hline Ga41As41H72 & 24.140 & 56.939 & 0.484 & 1.259 & 15.060 & 36.630 & 0.436 & 1.142 \\
\hline Protein & 62.970 & 77.188 & 1.368 & 1.728 & 37.111 & 44.554 & 1.104 & 1.338 \\
\hline QCD & 78.990 & 77.372 & 1.887 & 1.887 & 43.910 & 42.342 & 1.456 & 1.376 \\
\hline Si41Ge41H72 & 26.485 & 56.451 & 0.528 & 1.259 & 16.774 & 37.165 & 0.491 & 1.142 \\
\hline Wind Tunnel & 73.236 & 73.993 & 1.588 & 1.603 & 41.143 & 42.981 & 1.254 & 1.267 \\
\hline \multirow[t]{2}{*}{ Harmonic Mean } & 33.353 & $1.57 x$ & & & 21.235 & $1.52 x$ & & \\
\hline & \multicolumn{4}{|c|}{ Single Precision } & \multicolumn{4}{|c|}{ Double Precision } \\
\hline
\end{tabular}

Table 15. Incremental performance for the adaptivity technique on the regular benchmarks.

speedup for single-precision (double-precision) SpMV computation. As we can see (highlighted and in bold), most of the regular matrices can benefit from an improved memory footprint efficiency (which is measured as an increase in the quality metric $\left.q_{\left[b n \times b m, k_{\max }\right]}\right)$. A notable example is the matrix Circuit where the performance improves by more than a factor of 2 over its baseline without adaptivity. The best absolute performance is associated with the matrix Dense. Not surprisingly, AdELL+ provides a sufficient number of warps $n_{w}$ to achieve full utilization. On the other hand, there are examples in which adaptivity does not add any benefit. Consider the special case of the matrix Epidemiology. Its structure is composed of rows with an average of $\mu=4$ and almost zero variability. As a result, there is virtually no room for improvement and it is advisable to not apply the adaptivity technique in order to not to incur any implementation overhead (e.g. segmented reduction) on the performance. Fortunately, this feature can be easily integrated into AdELL+ by adding a flag $b_{3}$ into Flags [] to select between the two options.

\section{Putting it all together}

To recap, we designed AdELL+, an advanced ELL-based sparse matrix format that addresses the performance bottlenecks of the SpMV kernel. We tailored the underlying data structure on warp-granularity in order to suit the vectorized GPU hardware architecture. We introduced nonzero unrolling to optimize the memory hierarchy utilization. We mitigated the bandwidth-limited nature of the SpMV kernel by reducing the matrix memory footprint with blocking and indexing compression. Finally, we coped with matrix irregularity using adaptivity and our warp-balancing heuristic. We still have not analyzed and addressed the additional complexity associated with preprocessing. Indeed, selecting the best-tuned parameters may represent a practical issue when we use AdELL+ for real-world application. Therefore, we dedicate the next section to efficient (and effective) parameter auto-tuning.

\section{Online auto-tuning}

Parameter tuning is a key step to achieving the best possible performance from any advanced sparse matrix format. The overhead of evaluating points in the optimization parameter search space is determined by preprocessing and by actual kernel execution on the GPU hardware. In general, it is desirable to keep this one-time cost as small as possible in order for it to be amortizable over a reasonable number of SpMV iterations. This, in turn, means avoiding an exhaustive search by pruning the parameter space with some 


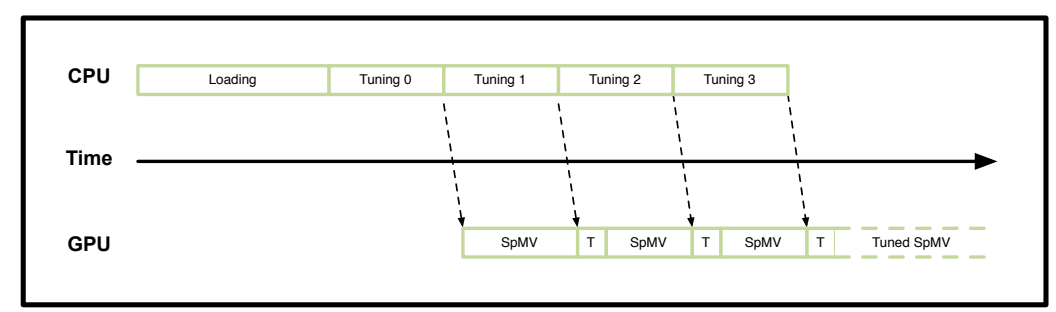

Fig. 9. Online auto-tuning CPU/GPU timeline.

clever strategy. Here we propose an online auto-tuning approach for AdELL+ that uses the quality metric $q_{\left[b n \times b m, k_{\max }\right]}$ to drive the process. The main novelty of this approach is the idea to tune while doing useful SpMV computation (as opposed to an initial offline tuning phase), completely hiding all the preprocessing overhead associated with composing AdELL+. The proposed auto-tuning schema is depicted in Figure 9. We have a timeline with CPU (where each tuning configuration is composed) and GPU (where it can be actually benchmarked). After loading the sparse matrix from the disk, the CPU processes the sparse format data structure and transfers it to the GPU. From that moment, the SpMV execution (e.g. a sparse solver) can begin. We can evaluate the performance by simply timing the SpMV kernel within the application context. In the mean time, the CPU is available to compose the second tuning configuration. Let us assume that the GPU has enough memory to contain two differently-tuned sparse matrices. Let us also assume that computing and data transfer can overlap (as in Kepler architecture [36]). The GPU can keep executing the original SpMV kernel until the entire second configuration has been transferred. Then, it can switch the computation to this latter for few iterations, evaluating the performance. Depending on which configuration is faster, we will keep one and discard the other. This process can be repeated as many times as needed on multiple configurations, providing an incremental online improvement towards the best-tuned configuration.

The proposed auto-tuning schema can be concretely applied to AdELL+ by introducing few additional considerations. We first analyze the preprocessing timing data reported in Table 16. The experimental computing platform on which those data have been generated was a dual-socket system with two 8-cores INTEL Xeon E5-2650@2GHz and 64GB@1.6GHz DDR3 memory. The operating system was 64-bit CentOS 6.6 with kernel 2.6.32-504.16.2. The compiler used was gcc 4.4.7. All the timings have been obtained as average over 10 runs. The first column of the Table 16 reports the time necessary to load the original Matrix Market file [33] from disk. The following columns are instead the preprocessing times to compose and tune CSR, AdELL+ and BCCOO [28]. Note that the measurements for this latter format are available only in single-precision due to the lack of double-precision implementation. Note also that using CSR we can draw a direct comparison with CSR+ [29] and CSR-Adaptive [30]. As we can see, the typical time to compose the AdELL+ data structure from an intermediate representation is $2.84 \mathrm{x}$ ( $2.57 \mathrm{x}$ for double-precision) higher than the CSR baseline. Indeed, the design choices associated with the warp-balancing heuristic have led to an overhead (i.e. first tuning configuration) that can be reasonably amortized. Second, we define the parameter space including all the $[b n \times b m]$ block factors used in Section 5 and all the $k_{\max }$ factors used in Section 7 (plus $k_{\max }=0$ to indicate no adaptivity applied). The nonzero unrolling technique is implemented by the means of differently-coded SpMV kernels applied on the same data structure. We empirically determined that $5 \mathrm{SpMV}$ executions are enough to get a performance evaluation for each unrolling factor lu. Hence, $25 \mathrm{SpMV}$ iterations (within the context of our online auto-tuning approach) are sufficient to find the best $l u$ associated with any new tuning configuration just transferred to GPU. The optimal CUDA block size is instead selected using the strategy outlined in Section 4. The goal is now to use the quality metric $q^{\left[b n \times b m, k_{\max }\right]}$ to prune the search space. We have already described the strong correlation between performance and quality metric. Hence, we may decide to evaluate $q^{\left[b n \times b m, k_{\max }\right]}$ for all the search space and pick the top-ranked configurations to be benchmarked on the GPU. Unfortunately, evaluating $q^{\left[b n \times b m, k_{\max }\right]}$ cannot be done without explicitly building the entire AdELL+ data structure. Note, $q_{[b n \times b m]}$ can approximate $q_{\left[b n \times b m, k_{\text {max }}\right]}$ due its ability to identify the most suitable blocking factors for a sparse matrix. In addition, $q_{[b n \times b m]}$ can be efficiently calculated without the need of composing AdELL+ (i.e. only its accessory data 
/ Journal of Parallel and Distributed Computing 00 (2015) 1-32

\begin{tabular}{|c|c|c|c|c|c|c|c|c|}
\hline \multirow[b]{3}{*}{ Matrix } & \multicolumn{8}{|c|}{ Time $[\mathrm{s}]$} \\
\hline & \multirow[b]{2}{*}{ Load } & CSR & \multicolumn{2}{|c|}{ AdELL+ } & \multirow{2}{*}{$\begin{array}{l}\text { BCCOO } \\
\text { Autotune }\end{array}$} & CSR & \multicolumn{2}{|c|}{ AdELL+ } \\
\hline & & \multicolumn{2}{|c|}{ Compose } & Autotune & & \multicolumn{2}{|c|}{ Compose } & Autotune \\
\hline Circuit & 2.624 & 0.071 & 0.410 & 0.278 & 2.098 & 0.082 & 0.395 & 0.328 \\
\hline Dense & 7.290 & 0.455 & 0.406 & 0.752 & 3.914 & 0.420 & 0.432 & 0.637 \\
\hline Economics & 3.683 & 0.098 & 0.576 & 0.343 & 2.397 & 0.113 & 0.558 & 0.222 \\
\hline Epidemiology & 5.345 & 0.104 & 0.745 & 0.314 & 3.448 & 0.108 & 0.713 & 0.316 \\
\hline FEM/Accelerator & 4.250 & 0.199 & 0.615 & 0.410 & 2.505 & 0.229 & 0.740 & 0.419 \\
\hline FEM/Cantilever & 6.652 & 0.209 & 0.216 & 0.530 & 2.335 & 0.317 & 0.441 & 0.531 \\
\hline FEM/Harbor & 4.053 & 0.192 & 0.465 & 0.353 & 1.582 & 0.261 & 0.690 & 0.355 \\
\hline FEM/Ship & 11.784 & 0.441 & 0.534 & 0.912 & 3.483 & 0.667 & 0.528 & 0.966 \\
\hline FEM/Spheres & 9.572 & 0.353 & 0.278 & 0.749 & 3.084 & 0.476 & 0.279 & 0.769 \\
\hline Ga41As41H72 & 29.918 & 1.501 & 4.452 & 2.675 & 14.379 & 1.699 & 4.436 & 2.712 \\
\hline Protein & 6.929 & 0.276 & 0.304 & 0.571 & 2.099 & 0.319 & 0.548 & 0.589 \\
\hline QCD & 3.107 & 0.173 & 0.086 & 0.278 & 1.438 & 0.218 & 0.111 & 0.300 \\
\hline $\mathrm{Si} 41 \mathrm{Ge} 41 \mathrm{H} 72$ & 24.747 & 1.257 & 3.494 & 2.306 & 11.479 & 1.411 & 3.473 & 2.211 \\
\hline Wind Tunnel & 18.538 & 0.522 & 1.099 & 1.307 & 4.750 & 0.605 & 1.130 & 1.228 \\
\hline Circuit5M & 171.222 & 4.527 & 16.442 & 9.831 & 53.024 & 5.315 & 16.423 & 9.207 \\
\hline Eu-2005 & 32.436 & 1.635 & 6.008 & 2.408 & 15.946 & 1.816 & 5.923 & 2.226 \\
\hline In-2004 & 28.160 & 1.204 & 5.304 & 1.998 & 12.305 & 1.363 & 5.191 & 2.284 \\
\hline LP & 21.431 & 2.026 & 2.253 & 2.694 & 51.349 & 2.033 & 3.077 & 2.779 \\
\hline Mip1 & 11.436 & 0.719 & 0.787 & 1.221 & 4.065 & 0.851 & 0.992 & 1.594 \\
\hline Webbase & 8.035 & 0.242 & 1.607 & 0.484 & 4.800 & 0.257 & 1.547 & 0.554 \\
\hline Arithmetic Mean & & 0.810 & $2.84 x$ & 1.658 & $6.04 x$ & 0.928 & $2.57 x$ & 1.969 \\
\hline & & \multicolumn{4}{|c|}{ Single Precision } & \multicolumn{3}{|c|}{ Double Precision } \\
\hline
\end{tabular}

Table 16. Preprocessing time for CSR and AdELL+.

structure). Table 16 reports the time necessary to autotune $q_{[b n \times b m]}$ for all the considered blocking factors using an OpenMP [43] implementation (indeed, each $q^{[b n \times b m]}$ can be calculated independently). Roughly, this is about $6 \mathrm{x}$ faster than tuning BCCOO. We conjecture that $q^{[b n \times b m]}$ can be also calculated while parsing the sparse matrix from disk, completely hiding any additional cost (the loading times, as reported in Table 16, are large enough to implement this strategy). Once we pick a subset of top-ranked blocking factors, we can iteratively benchmark all the combinations with $k_{\max }$. Alternatively, we can exploit the conceptual independency between blocking and adaptivity to reduce the search space, applying the line search strategy outlined in Table 17. First, we optimize $k_{\max }$ by testing all the feasible possibilities associated with the best blocking factor. In the given example, $[2 \times 1]$ is the top-ranked factor so we explore its $\left[2 \times 1, k_{\max }\right]$ combinations with $k_{\max }$ from $\infty$ (no bound) to 64 (we omit $k_{\max }=128$ because FEM/Harbor does not have any blocked row with more than 64 nonzero blocks). Second, we optimize $[b n \times b m, 16]$ by exploring the other top-ranked blocking factors (i.e. $[1 \times 2],[3 \times 1],[2 \times 2],[1 \times 3])$. As a result, we identify $[2 \times 1,16]$, the best-tuned configuration with 54.889 GFLOPS.

\begin{tabular}{|c||c|c|c|c|c|}
\hline \multicolumn{1}{|c||}{} & \multicolumn{5}{|c|}{ Performance [GFLOPS] } \\
\hline$k_{\max }$ & {$[2 \times 1]$} & {$[1 \times 2]$} & {$[3 \times 1]$} & {$[2 \times 2]$} & {$[1 \times 3]$} \\
\hline \hline$\infty$ & $\mathbf{4 9 . 7 1 5}$ & 46.239 & 46.012 & 42.318 & 43.550 \\
\hline 0 & $\mathbf{3 6 . 2 5 1}$ & 33.138 & 40.004 & 35.676 & 40.004 \\
\hline 4 & $\mathbf{4 5 . 7 8 0}$ & 40.595 & 44.495 & 45.864 & 41.031 \\
\hline 8 & $\mathbf{5 2 . 9 0 0}$ & 47.350 & 51.961 & 51.725 & 44.043 \\
\hline 16 & $\mathbf{5 4 . 8 8 9}$ & $\mathbf{4 8 . 2 1 8}$ & $\mathbf{5 2 . 2 6 8}$ & $\mathbf{5 0 . 3 1 9}$ & $\mathbf{4 3 . 4 1 1}$ \\
\hline 32 & $\mathbf{5 2 . 8 1 0}$ & 46.245 & 49.943 & 47.428 & 43.631 \\
\hline 64 & $\mathbf{4 9 . 7 7 1}$ & 46.268 & 46.029 & 45.701 & 43.482 \\
\hline 128 & 49.677 & 46.209 & 45.971 & 42.436 & 43.633 \\
\hline \hline & \multicolumn{5}{|c|}{ Single Precision } \\
\hline
\end{tabular}

Table 17. FEM/Harbor parameter tuning with line search. 


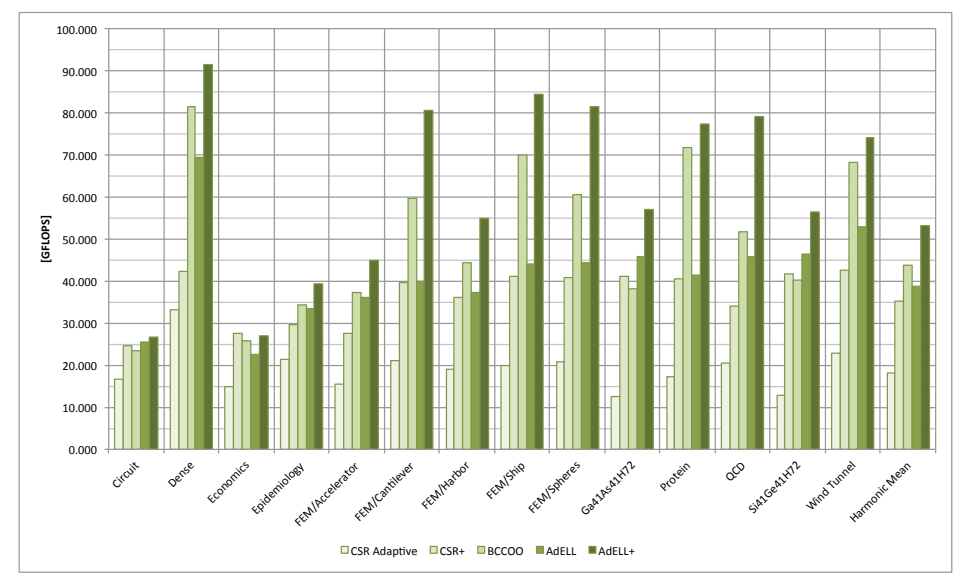

Fig. 10. AdELL+ single-precision performance on the regular benchmarks.

\section{Comparison with the state-of-the-art}

In this section, we provide a performance-focused comparison of auto-tuned AdELL+ with other advanced sparse matrix formats. Specifically, we considered BCCOO [28] (which is arguably the state-ofthe-art in SpMV optimization), CSR+ [29] and CSR-Adaptive [30]. Regarding the first two sparse formats, we used the code made available by their respective authors. Regarding CSR-Adaptive, we used the implementation contained in the linear algebra library ViennaCL [44]. In addition, we compared AdELL+ with our previous work AdELL [25] (i.e. turning off blocking, index compression and upper limit $k_{\max }$ for the warp-balancing heuristic). Our experimental results on Tesla K40 for single-precision computation are illustrated in Figure 10 and 11. As we can observe, AdELL+ consistently achieves comparable or better performance that the state-of-the-art. On the regular benchmark suite, AdELL+ has a 1.22x speedup over BCCOO, a 1.52x over CSR+, a 2.95x over CSR-Adaptive and a 1.38x over AdELL. AdELL+ performs better on each individual matrix but Economics, where its performance is comparable with CSR + . Indeed, these results are directly correlated with the SIMD-oriented layout and the nonzero unrolling that leverage the GPU hardware at its best. As an aside note, the ViennaCL implementation of CSR-Adaptive does not perform too well on the the NVIDIA Kepler architecture, at least when compared with the results reported by Greathouse and Daga [30] on a comparable AMD GPU. On the irregular benchmark suite, AdELL+ still has an edge over the competition. Specifically, we measured a 1.05x speedup over BCCOO, a 1.16x over CSR+, a 2.28x over CSR-Adaptive and a 1.89x over AdELL. Perhaps the reduced speedup is due to

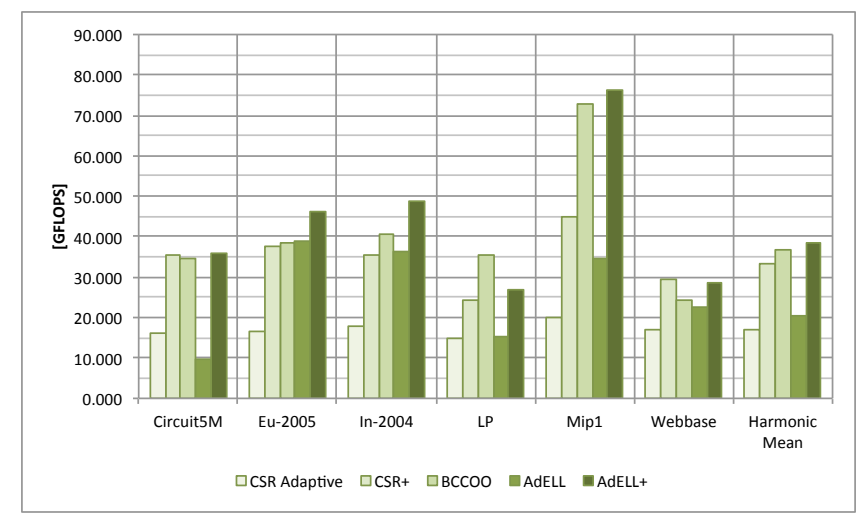

Fig. 11. AdELL+ single-precision performance on the irregular benchmarks. 


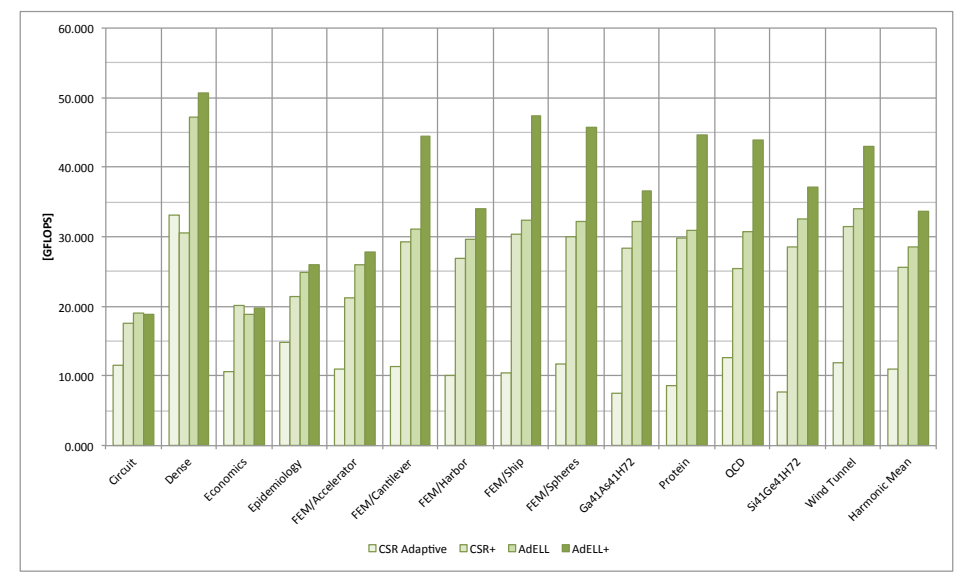

Fig. 12. AdELL+ double-precision performance on the regular benchmarks.

the fact that both BCCOO and CSR+ do a good job already in terms of load balancing. Moreover, there is very little structure in terms of dense subblocks and nonzero patterns to leverage for compression. Last, we can notice that BCCOO clearly obtains the best performance for matrix LP. This particular benchmark has only 4284 rows associated with 54743 atomic warps that create a high level of conflict when reducing the result to vector $\mathbf{y}$. Although BCCOO obtained its advantage using a non-portable synchronization-free segmented reduction, we conjecture that the problem can be mitigated by implementing a reduction strategy more similar to the one in CSR+. In Section 2.2, we estimated a 51.88 GFLOPS theoretical single-precision performance peak associated with ELL-based formats. AdELL+ breaks this limit for the regular benchmark suite (53.323 GFLOPS) whereas it achieves a fairly high peak portion (38.628 GFLOPS) for the irregular matrix suite. The former result (even surpassing the theoretical peak) comes from the use of blocking and delta-based compression. Note also that AdELL+ provides a substantial improvement over AdELL for both regular and irregular benchmarks, justifying the additional optimization techniques.

Figure 12 and 13 reports the same computational tests in double-precision. Again, we could not include BCCOO due to the lack of double-precision implementation. As we can see, AdELL+ still consistently outperforms the other advanced sparse matrix formats. On the regular benchmark suite, AdELL+ is the best for all the matrices with an overall 1.31x speedup over CSR+, a 3.08x over CSR-Adaptive and a 1.18x over AdELL. On the irregular benchmarks, AdELL+ still achieves comparable or better performance with a unique exception of the matrix Webbase (where AdELL+ is only slightly behind CSR+). Again, we conjecture that a little variation in the atomic segmented reduction mechanism may bridge the performance

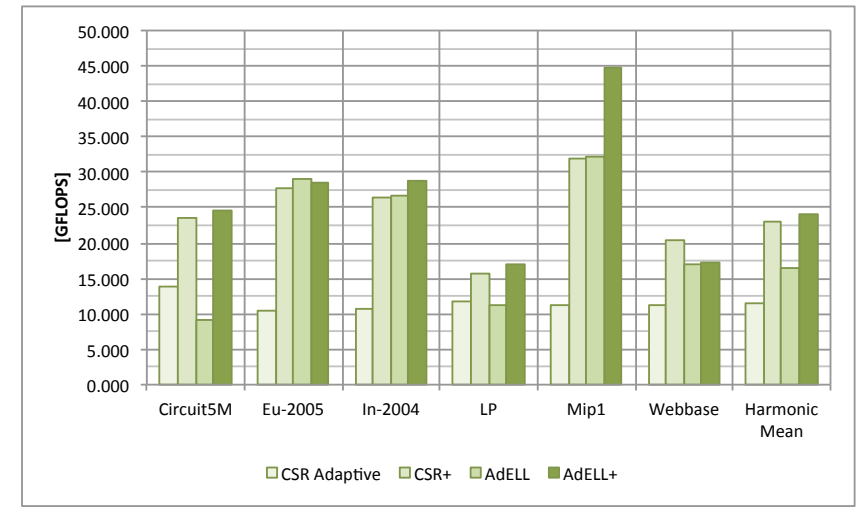

Fig. 13. AdELL+ double-precision performance on the irregular benchmarks. 


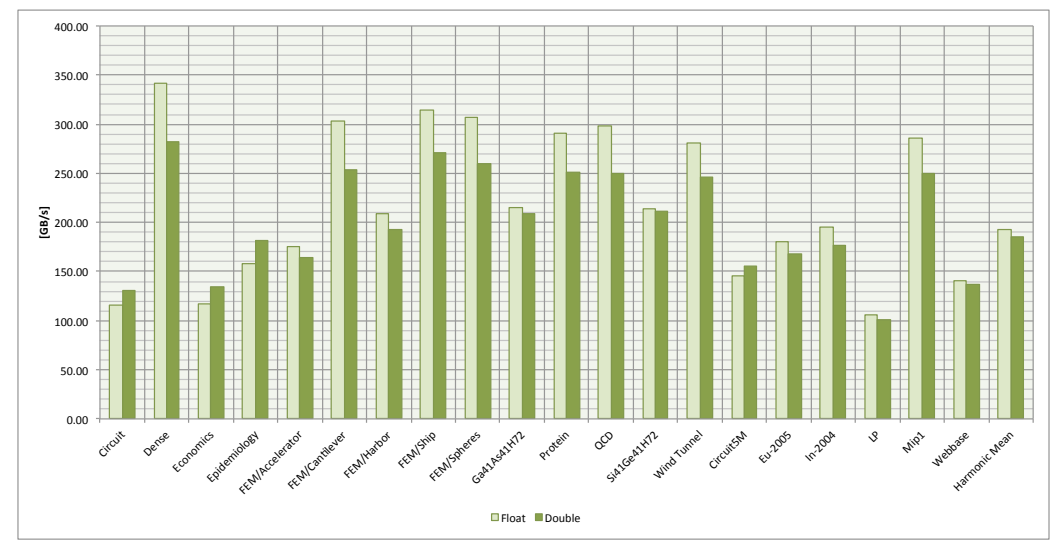

Fig. 14. AdELL memory bandwidth.

gap. For double-precision, we estimated a 34.58 GFLOPS theoretical performance peak. This time AdELL+ does not break the limit for regular matrices but it comes very close (33.697 GFLOPS). On the other hand, AdELL+ still achieves a fairly high peak portion (24.067 GFLOPS) for irregular matrices.

\section{Memory Considerations}

We have seen how SpMV is a bandwidth-bound kernel. In this section, we are interested in analyzing the relationship between performance and GPU memory hierarchy. Table 14 reports the effective memory bandwidth of the best-tuned AdELL+ kernel for all the benchmarks. Intuitively, memory bandwidth results close to the $207.5 \mathrm{~GB} / \mathrm{s}$ peak indicate that the AdELL+ kernel is efficiently using the underlying GPU memory resources. We observe that some of the benchmarks (e.g. FEM/Ship) have an effective bandwidth higher than the measured peak. Indeed, this is an artifact of blocking and compression. On the other hand,

\begin{tabular}{|c||r||r|r|c|}
\hline \multicolumn{1}{|c||}{} & \multicolumn{2}{c|}{ [GFLOPS] } & \multicolumn{2}{c|}{ Hit Rate } \\
\cline { 2 - 5 } & w/o & w/ & Texture & L2 \\
\hline \hline Circuit & 14.406 & 19.486 & 0.699 & 0.616 \\
\hline Dense & 39.711 & 43.825 & 0.970 & 0.983 \\
\hline Economics & 113.558 & 21.407 & 0.676 & 0.908 \\
\hline Epidemiology & 24.568 & 25.018 & 0.597 & 0.690 \\
\hline FEM/Accelerator & 16.551 & 28.433 & 0.623 & 0.839 \\
\hline FEM/Cantilever & 32.887 & 39.113 & 0.866 & 0.945 \\
\hline FEM/Harbor & 28.131 & 33.831 & 0.861 & 0.917 \\
\hline FEM/Ship & 32.853 & 38.018 & 0.857 & 0.909 \\
\hline FEM/Spheres & 28.445 & 37.417 & 0.892 & 0.857 \\
\hline Ga41As41H72 & 27.109 & 37.620 & 0.686 & 0.890 \\
\hline Protein & 30.021 & 35.964 & 0.870 & 0.964 \\
\hline QCD & 29.038 & 35.257 & 0.760 & 0.872 \\
\hline Si41Ge41H72 & 126.986 & 38.123 & 0.917 & 0.691 \\
\hline Wind Tunnel & 34.535 & 38.474 & 0.541 & 0.932 \\
\hline \hline Circuit5M & 6.648 & 9.081 & 0.158 & 0.786 \\
\hline Eu-2005 & 21.083 & 29.594 & 0.820 & 0.779 \\
\hline In-2004 & 23.370 & 28.135 & 0.702 & 0.863 \\
\hline LP & 11.252 & 11.093 & 0.132 & 0.310 \\
\hline Mip1 & 23.203 & 29.385 & 0.619 & 0.940 \\
\hline Webbase & 15.230 & 16.948 & 0.528 & 0.712 \\
\hline Harmonic Mean & 19.803 & $\mathbf{1 . 2 6 x}$ & & \\
\hline
\end{tabular}

Table 18. Cache analysis for AdELL+. 
some benchmarks (e.g. LP) underperform due to a very irregular access to the dense vector $\mathbf{x}$. In our computational experiments, we observed that texture cache is currently the best approach to mitigate this irregularity. We considered the AdELL+ kernel with no blocking and upper limit (i.e. $[1 \times 1, \infty])$ and we measured the performance with and without read-only memory. In addition, we collected the hit rate for L2 and texture cache. Those results are reported in Table 18. As we can see, texture cache provides an immediate (and effortless) way to improve performance by an average factor of $1.26 \mathrm{x}$. We can also observe how $\mathrm{L} 2$ cache achieves an average hit rate of $63.77 \%$. On the other hand, texture cache has a lower hit rate due to its limited size of only $48 \mathrm{~KB}$ per SMX (as opposed to $1536 \mathrm{~KB}$ for the coherent L2 cache). Locality may be improved by slicing the matrix into vertical portions as wide as the number of elements that fit into cache memory. However, this technique has the drawback of requiring separate SpMV kernel launches, one for each slice. Another technique known as vector expansion [45] copes with irregularity by expanding the access pattern over a dense array with $n n z$ elements. This, in turn, leads to a fully coalesced access during the SpMV kernel. However, we conjecture that composing the expanded vector is as expensive as the irregular memory pattern itself, making the overall approach equivalent to the original SpMV kernel. Indeed, this technique is limited to the case in which the dense vector $\mathbf{x}$ does not change and, hence, can be reused over and over. Last, we are also interested in analyzing the memory footprint associated with the besttuned AdELL+. Table 19 provides a comparison with CSR taken as baseline for its well-known compact representation. In general, AdELL+ provides a more compact memory footprint measured as a $0.86 \mathrm{x}$ ratio for single-precision representation and as a $0.93 \mathrm{x}$ ratio for double-precision representation. This reduction is primarily due to the blocking and delta-based index compression techniques, and secondarily to adaptivity (which arranges nonzero more efficiently across the warps). On the irregular matrices, the compression has in general less room for improvement. Hence, AdELL+ cannot always be more compact than CSR. This also provides an additional explanation regarding the double-precision performance on matrix Webbase. As we can see, AdELL+ has a slightly bigger memory footprint than CSR (44.92MB vs 39.35MB) which can be correlated with the slightly inferior SpMV performance.

\begin{tabular}{|c||r|r||r|r|}
\hline \multicolumn{1}{|c||}{} & \multicolumn{4}{|c|}{ Memory Footprint [MB] } \\
\cline { 2 - 5 } & CSR & AdELL+ & CSR & AdELL+ \\
\hline \hline Circuit & 7.97 & 8.63 & 11.63 & 13.00 \\
\hline Dense & 30.53 & $\mathbf{1 5 . 7 6}$ & 45.78 & $\mathbf{3 1 . 0 4}$ \\
\hline Economics & 10.50 & 10.64 & 15.36 & 16.65 \\
\hline Epidemiology & 18.03 & 18.16 & 26.04 & $\mathbf{2 2 . 2 7}$ \\
\hline FEM/Accelerator & 20.48 & $\mathbf{1 8 . 4 8}$ & 30.50 & 31.73 \\
\hline FEM/Cantilever & 30.81 & $\mathbf{1 7 . 5 3}$ & 46.10 & $\mathbf{3 4 . 3 7}$ \\
\hline FEM/Harbor & 18.29 & $\mathbf{1 4 . 0 3}$ & 27.35 & $\mathbf{2 4 . 9 9}$ \\
\hline FEM/Ship & 60.15 & $\mathbf{3 3 . 6 8}$ & 89.95 & $\mathbf{6 4 . 9 5}$ \\
\hline FEM/Spheres & 46.17 & $\mathbf{2 6 . 1 3}$ & 69.10 & $\mathbf{5 0 . 7 3}$ \\
\hline Ga41As41H72 & 142.08 & $\mathbf{1 1 2 . 5 0}$ & 212.61 & $\mathbf{1 8 5 . 8 1}$ \\
\hline Protein & 33.29 & $\mathbf{1 9 . 2 4}$ & 49.86 & $\mathbf{3 7 . 2 1}$ \\
\hline QCD & 14.81 & $\mathbf{7 . 7 6}$ & 22.13 & $\mathbf{1 5 . 0 7}$ \\
\hline Si41Ge41H72 & 115.24 & $\mathbf{9 1 . 3 8}$ & 172.50 & $\mathbf{1 5 0 . 8 6}$ \\
\hline Wind Tunnel & 89.59 & $\mathbf{5 5 . 5 5}$ & 133.98 & $\mathbf{1 0 5 . 2 5}$ \\
\hline \hline Circuit5Ml & 475.34 & 481.20 & 702.41 & $\mathbf{6 9 6 . 7 5}$ \\
\hline Eu-2005 & 150.04 & 151.20 & 223.42 & $\mathbf{2 3 3 . 1 9}$ \\
\hline In-2004 & 134.34 & $\mathbf{1 1 8 . 9 2}$ & 198.88 & 207.85 \\
\hline LP & 86.07 & $\mathbf{8 1 . 1 0}$ & 129.10 & 134.62 \\
\hline Mip1 & 79.24 & $\mathbf{5 0 . 7 2}$ & 118.73 & $\mathbf{9 3 . 6 9}$ \\
\hline Webbase & 27.51 & 31.84 & 39.35 & 44.92 \\
\hline \hline Arithmetic Mean & 79.52 & $\mathbf{0 . 8 6} x$ & 118.24 & $\mathbf{0 . 9 3 x}$ \\
\hline \hline & Single Precision & Double Precision \\
\hline
\end{tabular}

Table 19. Memory footprint. 


\section{Conclusions}

Our research work was aimed to improve the state-of-the-art on SpMV optimization on GPUs. After analyzing the performance bottleneck of sparse linear algebra computation, we proposed to combine several optimization techniques into an efficient ELL-based sparse format named AdELL+. We first provided a foundation for AdELL+ based on a SIMD-oriented memory and computational layout (warp granularity) that suit the underlying GPU hardware architecture. We addressed the bandwidth-limited nature of the SpMV kernel with the blocking and delta-based index compression technique. Moreover, we improved the memory hierarchy efficiency with nonzero unrolling. We coped with matrix irregularity using balanced warps composed using a parametrized warp-balancing heuristic. Finally, we introduced a novel online autotuning approach that uses a quality metric to predict efficient block factors and progressively explores the parameter space while useful SpMV computation is done (hiding preprocessing overhead). Our experimental results provided substantial evidence that AdELL+ outperforms other state-of-the-art sparse formats proposed in academia (BCCOO) and industry (CSR+ and CSR-Adaptive). Moreover, our auto-tuning approach makes AdELL+ viable for real-world applications.

Sparse linear algebra is a powerful framework used in numerous areas of science and engineering. Optimizing the SpMV kernel is surely critical to solve today's computation problems faster and to determining which problems we will be able to solve tomorrow. This motivated us (as well as many other researchers) to pour a significant effort into implementations based on modern-day parallel computer architectures. We believe that the performance improvement associated with AdELL+ is a significant and important result since it is an improvement over an already outstanding body of previous works in SpMV optimization. We were also able to ground the presented optimization techniques on higher-level theoretical considerations (i.e. quality metric). As a result, the ideas presented in this paper are general enough to be valid for future many-core GPU architectures and produce a long-lasting impact. We are also confident that the adoption of AdELL+ will simplify the life of sparse linear algebra library users, relieving from the burden to explore among different sparse matrix formats. Indeed, AdELL+ (along with its auto-tuning approach) consistently provides a one-size-fits-all SpMV performance that is the best (or, at least, a very good one) over heterogenous application domains.

\section{Acknowledgments}

This work was supported by NSF grants IIS-106468. We would also like to thank Nvidia for donating the Testa K40 GPU used for our performance tests.

\section{References}

[1] K. Asanovic, R. Bodik, B. C. Catanzaro, J. J. Gebis, P. Husbands, K. Keutzer, D. A. Patterson, W. L. Plishker, J. Shalf, S. W. Williams, K. A. Yelick, The Landscape of Parallel Computing Research: A View from Berkeley, Tech. rep., EECS Department, University of California, Berkeley (Dec 2006).

URL http: //www . eecs . berkeley.edu/Pubs/TechRpts/2006/

[2] Y. Saad, Iterative Methods for Sparse Linear Systems, 2nd Edition, Society for Industrial and Applied Mathematics, 2003.

[3] S. Mehrotra, On the Implementation of a Primal-Dual Interior Point Method, SIAM Journal on Optimization 2 (4) (1992) 575601.

URL http: //epubs.siam.org/doi/abs/10.1137/0802028

[4] E. J. Im, K. Yelick, Optimization of Sparse Matrix Kernels for Data Mining, in: SIAM Conference on Data Mining, 2000.

[5] J. R. Gilbert, S. Reinhardt, V. B. Shah, High-performance Graph Algorithms from Parallel Sparse Matrices, in: International Conference on Applied Parallel Computing (PARA), 2007, pp. 260-269.

URL http://dl .acm.org/citation.cfm?id=1775059.1775097

[6] J. Davis, E. Chung, SpMV: A Memory-Bound Application on the GPU Stuck between a Rock and a Hard Place, Tech. rep., Microsoft (2012).

[7] S. Huang, S. Xiao, W. Feng, On the Energy Efficiency of Graphics Processing Units for Scientific Computing, in: Proceedings of the IEEE International Symposium on Parallel and Distributed Processing (IPDPS), 2009, pp. 1-8. URL http://dx.doi.org/10.1109/IPDPS. 2009.5160980

[8] S. Williams, L. Oliker, R. Vuduc, J. Shalf, K. Yelick, J. Demmel, Optimization of Sparse Matrix-Vector Multiplication on Emerging Multicore Platforms, in: Proceedings of the ACM/IEEE Conference on High Performance Computing Networking, Storage and Analysis (SC), 2007, pp. 38:1-38:12.

URL http://doi.acm.org/10.1145/1362622.1362674 
[9] N. Bell, M. Garland, Implementing Sparse Matrix-Vector Multiplication on Throughput-Oriented Processors, in: Proceedings of the ACM/IEEE Conference on High Performance Computing Networking, Storage and Analysis (SC), 2009, pp. 18:1-18:11. URL http://doi .acm.org/10.1145/1654059.1654078

[10] NVIDIA, CUDA Sparse Matrix Library cuSPARSE.

URL http://docs.nvidia.com/cuda/cusparse/

[11] F. Vázquez, J. J. Fernández, E. M. Garzón, A New Approach for Sparse Matrix Vector Product on NVIDIA GPUs, Concurrency and Computation: Practice and Experience 23 (8) (2011) 815-826. URL http://dx.doi.org/10.1002/cpe.1658

[12] M. M. Baskaran, R. Bordawekar, Optimizing Sparse Matrix-Vector Multiplication on GPUs, Tech. rep., IBM (2009).

[13] A. Monakov, A. Lokhmotov, A. Avetisyan, Automatically Tuning Sparse Matrix-Vector Multiplication for GPU Architectures, in: High Performance Embedded Architectures and Compilers, Vol. 5952, Springer Berlin Heidelberg, 2010, pp. 111-125. URL http://dx.doi.org/10.1007/978-3-642-11515-8_10

[14] M. Kreutzer, G. Hager, G. Wellein, H. Fehske, A. R. Bishop, A Unified Sparse Matrix Data Format for Modern Processors with Wide SIMD units, CoRR 2013. URL http: //arxiv.org/abs/1307.6209

[15] J. W. Choi, A. Singh, R. W. Vuduc, Model-driven Autotuning of Sparse Matrix-vector Multiply on GPUs, in: Proceedings of the ACM SIGPLAN Symposium on Principles and Practice of Parallel Programming (PPoPP), 2010, pp. 115-126. URL http://doi .acm.org/10.1145/1693453.1693471

[16] J. C. Pichel, F. F. Rivera, M. Fernández, A. Rodríguez, Optimization of Sparse Matrix-Vector Multiplication Using Reordering Techniques on GPUs, Microprocessors and Microsystems 36 (2) (2012) 65-77. URL http://dx.doi.org/10.1016/j.micpro.2011.05.005

[17] W. T. Tang, W. J. Tan, R. Ray, Y. W. Wong, W. Chen, S.-h. Kuo, R. S. M. Goh, S. J. Turner, W.-F. Wong, Accelerating Sparse Matrix-vector Multiplication on GPUs Using Bit-Representation-Optimized Schemes, in: Proceedings of the ACM/IEEE Conference on High Performance Computing Networking, Storage and Analysis (SC), 2013, pp. 26:1-26:12. URL http://doi.acm.org/10.1145/2503210.2503234

[18] S. Xu, H. X. Lin, W. Xue, Sparse Matrix-Vector Multiplication Optimizations based on Matrix Bandwidth Reduction using NVIDIA CUDA, in: Proceedings of International Symposium on Distributed Computing and Applications to Business Engineering and Science (DCABES), 2010, pp. 609-614.

[19] B.-Y. Su, K. Keutzer, clSpMV: A Cross-Platform OpenCL SpMV Framework on GPUs, in: Proceedings of the ACM International Conference on Supercomputing (ICS), 2012, pp. 353-364. URL http://doi.acm.org/10.1145/2304576.2304624

[20] F. Vázquez, G. Ortega, J. Fernández, E. Garzón, Improving the Performance of the Sparse Matrix Vector Product with GPUs, in: Proceedings of IEEE International Conference on Computer and Information Technology (CIT), 2010, pp. $1146-1151$.

[21] X. Feng, H. Jin, R. Zheng, K. Hu, J. Zeng, Z. Shao, Optimization of sparse matrix-vector multiplication with variant csr on gpus, in: Proceedings of IEEE International Conference on Parallel and Distributed Systems (ICPADS), 2011, pp. 165-172.

[22] Z. Koza, M. Matyka, S. Szkoda, Ł. Mirosław, Compressed Multirow Storage Format for Sparse Matrices on Graphics Processing Units, SIAM Journal on Scientific Computing 36 (2) (2014) C219-C239. URL http://dx.doi .org/10.1137/120900216

[23] T. Oberhuber, A. Suzuki, J. Vacata, New Row-grouped CSR Format for Storing the Sparse Matrices on GPU with Implementation in CUDA, CoRR 2010. URL http://arxiv.org/abs/1012.2270

[24] M. Heller, T. Oberhuber, Adaptive Row-Grouped CSR Format for Storing of Sparse Matrices on GPU, CoRR 2012. URL http: //arxiv .org/abs/1203.5737

[25] M. Maggioni, T. Berger-Wolf, AdELL: An Adaptive Warp-Balancing ELL Format for Efficient Sparse Matrix-Vector Multiplication on GPUs, in: Proceedings of International Conference on Parallel Processing (ICPP), 2013, pp. 11-20.

[26] A. Ashari, N. Sedaghati, J. Eisenlohr, P. Sadayappan, An Efficient Two-dimensional Blocking Strategy for Sparse Matrix-vector Multiplication on GPUs, in: Proceedings of the ACM International Conference on Supercomputing (ICS), 2014 , pp. $273-282$. URL http://doi .acm.org/10.1145/2597652.2597678

[27] A. Ashari, N. Sedaghati, J. Eisenlohr, S. Parthasarathy, P. Sadayappan, Fast Sparse Matrix-vector Multiplication on GPUs for Graph Applications, in: Proceedings of the ACM/IEEE Conference on High Performance Computing Networking, Storage and Analysis (SC), 2014, pp. 781-792. URL http://dx.doi.org/10.1109/SC. 2014.69

[28] S. Yan, C. Li, Y. Zhang, H. Zhou, yaSpMV: Yet Another SpMV Framework on GPUs, in: Proceedings of the ACM SIGPLAN Symposium on Principles and Practice of Parallel Programming (PPoPP), 2014, pp. 107-118. URL http: //doi .acm.org/10.1145/2555243.2555255

[29] S. Baxter, Modern GPU, Code Intended to Promote New and Productive Ways of Thinking about GPU Computing. URL http: //nvlabs.github.io/moderngpu/

[30] J. L. Greathouse, M. Daga, Efficient Sparse Matrix-vector Multiplication on GPUs Using the CSR Storage Format, in: Proceedings of the International Conference for High Performance Computing, Networking, Storage and Analysis (SC), 2014, pp. 769-780. URL http://dx.doi.org/10.1109/SC.2014.68

[31] M. Maggioni, T. Berger-Wolf, CoAdELL: Adaptivity and Compression for Improving Sparse Matrix-Vector Multiplication on GPUs, in: Proceedings of the IEEE International Parallel Distributed Processing Symposium Workshops (IPDPSW), 2014, pp. 933-940.

[32] T. A. Davis, Y. Hu, The University of Florida Sparse Matrix Collection, ACM Transaction on Mathematical Software (TOMS) 
38 (1) (2011) 1:1-1:25.

URL http://doi.acm.org/10.1145/2049662.2049663

[33] R. F. Boisvert, R. Pozo, K. Remington, R. F. Barrett, J. J. Dongarra, Matrix Market: A Web Resource for Test Matrix Collections, in: Proceedings of the IFIP TC2/WG2.5 Working Conference on Quality of Numerical Software: Assessment and Enhancement, 1997, pp. 125-137.

URL http: //dl .acm.org/citation. cfm?id=265834.265854

[34] D. Langr, P. Tvrdik, Evaluation Criteria for Sparse Matrix Storage Formats, IEEE Transactions on Parallel and Distributed Systems PP (99) (2015) 1-14.

[35] S. Williams, A. Waterman, D. Patterson, Roofline: An Insightful Visual Performance Model for Multicore Architectures, Communications of the ACM 52 (4) (2009) 65-76.

URL http://doi .acm.org/10.1145/1498765.1498785

[36] NVIDIA, NVIDIA's Next Generation CUDA Compute Architecture: Kepler GK110.

URL http://www.nvidia.com/content/PDF/kepler/NVIDIA-Kepler-GK110-Architecture-Whitepaper.pdf

[37] E. Bardsley, A. Donaldson, Warps and Atomics: Beyond Barrier Synchronization in the Verification of GPU Kernels, in: NASA Formal Methods, Vol. 8430, Springer International Publishing, 2014, pp. 230-245.

URL http://dx.doi.org/10.1007/978-3-319-06200-6_18

[38] M. Maggioni, T. Berger-Wolf, J. Liang, GPU-Based Steady-State Solution of the Chemical Master Equation, in: Proceedings of the IEEE International Parallel Distributed Processing Symposium Workshops (IPDPSW), 2013, pp. 579-588.

[39] NVIDIA, CUDA: Compute Unified Device Architecture.

URL http://docs.nvidia.com/cuda/\#axzz3apxVEBRJ

[40] Khronos, OpenCL: The Open Standard for Parallel Programming of Heterogeneous Systems. URL https: //www. khronos.org/opencl/

[41] G. Murthy, M. Ravishankar, M. Baskaran, P. Sadayappan, Optimal Loop Unrolling for GPGPU Programs, in: Proceedings of the IEEE International Symposium on Parallel and Distributed Processing (IPDPS), 2010, pp. 1-11.

[42] J. Kleinberg, Y. Rabani, É. Tardos, Fairness in routing and load balancing, Journal of Computer and System Sciences 63 (1) (2001) 2-20. doi:http://dx.doi.org/10.1006/jcss.2001.1752.

URL http://www.sciencedirect.com/science/article/pii/S0022000001917520

[43] L. Dagum, R. Menon, OpenMP: An Industry-Standard API for Shared-Memory Programming, IEEE Computational Science and Engineering 5 (1) (1998) 46-55.

URL http://dx.doi.org/10.1109/99.660313

[44] K. Rupp, ViennaCL: A High Level Linear Algebra Library for GPUs and Multi-Core CPUs. URL http: //viennacl. sourceforge.net/

[45] Y. Deng, B. Wang, S. Mu, Taming irregular EDA applications on GPUs, in: IEEE/ACM International Conference on ComputerAided Design (ICCAD), 2009, pp. 539-546. 